\title{
Radio pulsar binaries in globular clusters: their orbital eccentricities and stellar interactions
}

\author{
Manjari Bagchi and Alak Ray \\ Tata Institute of Fundamental Research, Mumbai 400005, India
}

\begin{abstract}
High sensitivity searches of globular clusters (GC) for radio pulsars by improved pulsar search algorithms and sustained pulsar timing observations have so far yielded some 140 pulsars in more than two dozen GCs. The observed distribution of orbital eccentricity and period of binary radio pulsars in GCs have imprints of the past interaction between single pulsars and binary systems or of binary pulsars and single passing non-compact stars. It is seen that GCs have different groups of pulsars. These may have arisen out of exchange or merger of a component of the binary with the incoming star or a "fly-by" in which the original binary remains intact but undergoes a change of eccentricity and orbital period. We consider the genesis of the distribution of pulsars using analytical and computational tools such as STARLAB, which performs numerical scattering experiments with direct N-body integration. Cluster pulsars with intermediate eccentricities can mostly be accounted for by fly-bys whereas those with high eccentricities are likely to be the result of exchanges and/or mergers of single stars with the binary companion of the pulsar, although there are a few objects which do not easily fit into this description. The corresponding distribution for galactic field pulsars shows notable differences from the GC pulsar orbital period and eccentricity distribution. The long orbital period pulsars in the galactic field with frozen out low eccentricities are largely missing from the globular clusters, and we show that ionization of these systems in GCs cannot alone account for the peculiarities.
\end{abstract}

Subject headings: pulsars: general - globular clusters: general — stellar dynamics — methods: N-body simulations — binaries (including multiple): close

\section{Introduction}

Globular clusters (GCs) are dense spherical collection of stars orbiting around the galactic center containing a significant number of binary stars. The first millisecond radio pulsar 
was discovered in a GC M28 only in 1987 (Lvne et al. 1987) and GCs in general were regarded as spun-up pulsar nurseries (Alpar et al. 1982). How old and evolved stellar systems like GCs can contain apparently young, active objects like pulsars have led to suggestions, based on lifetime constraints from orbital decay by gravitational radiation (Prince et al 1991), that some of these systems have formed recently, or are forming even today. Interaction between binary stars and single stars in GCs is believed to be an important dynamical process as the resulting binary systems may provide a substantial source of energy for the GCs, as the binding energies of a few, very close binaries (like neutron star binaries) can approach that of a moderately massive host GC (Spitzer 1987; Hut et al. 2003). Although the importance there is due to exchange of energy, interesting constraints and conclusions can be derived about lifetimes of binaries from the rate at which their orbital eccentricity is changed by encounter with other stars. A key aspect here is that the orbital eccentricities of binary millisecond radio pulsars can be measured with extraordinary precision.

Pulsar spin-up and recycling are thought to be enhanced in globular clusters by binarysingle star interactions. This is because binary interactions can increase the cross section for recycling processes and weaken the stellar density dependence of the probability of a neutron star without visible radio pulses evolving into a reborn pulsar after the (binary) interaction (Sigurdsson \& Phinney 1993). After the interaction some of the pulsars may be ejected from the core of the cluster or even from the GC altogether, and their distributions of orbital properties can be predicted in terms of the interaction model. Thus, observable parameters of the neutron stars and their binary companions in globular clusters, such as spin, orbital period and eccentricity, projected radial position in the cluster, companion mass and their distributions provide a tracer of the past history of dynamical interactions of the binary NSs in individual GCs. These parameters can even provide a valuable test-bed to examine the theoretical scenarios of formation and evolution of recycled pulsars. Among the different types of neutron star binaries, binary radio pulsars can be timed more easily and accurately by ground-based telescopes over well-separated epochs, as the underlying neutron stars are less prone to noise than in the case of X-ray pulsars which have episodically varying accretion torques. These lead to the easier measurement of orbital parameters. Eccentric millisecond pulsar binaries can also be important probes of neutron star physics since they provide a way to constrain the masses of the fully-recycled pulsars. Pulsar timing can measure the orbital advance of the periastron of the elliptical orbit; this advance is dominated by general relativistic effects if the companion star is a compact one, and can determine the total mass of the system. The recycling scenario creates binary millisecond pulsars in circular orbits as during the accretion phase there is a strong tidal coupling leading to orbit circularization. Therefore, the eccentric systems of fully recycled (millisecond period) pulsar binaries found in globular clusters are produced only through stellar interactions where density of stars is 
high (Rasio \& Heggie 1995).

Till today, a total of 140 pulsars in 26 GCA 1 have been discovered. Among them 74 are known as binaries (in $23 \mathrm{GCs}$ ), 59 are known as isolated and 7 have no published timing/orbital solutions. In the present work, we concentrate on binaries. For one of them (PSR J2140-2310B in M30), orbital parameters are not well determined, only lower limit of $P_{\text {orb }}$ and $e$ are known as $P_{\text {orb }}>0.8$ days and $e>0.52$. So we exclude it from our analysis and the total number of pulsars in become 73 . We study the distribution of these 73 pulsars in the orbital eccentricity - period plane with analytical results and our numerical experiments of scattering of stars simulated by direct N-body integration tools. The corresponding distribution of some of these observables for neutron star binaries in the galactic field e.g. in the orbital eccentricity and period plane have some significant differences. We discuss below these differences in the light of interaction of stars with binaries already formed or in the process of formation inside globular clusters, due to the high stellar density in their cores compared to that of the galactic disk.

This article is in continuation of our preliminary work in this area (Bagchi \& Ray 2009) and provides further details and extensions. The present paper is organized as follows: in section 2 we discuss the characteristics of the presently available binary radio pulsar data and how they occupy different regions of the orbital phase space. Here we include both GC pulsars and disk pulsars as the later class was not considered in the earlier paper (Bagchi \& Ray 2009). Note that in Fig 1, 2 and 5, we plot the GC and galactic disk pulsars irrespective of their spins. Our conclusions are not significantly affected if we restrict the sample to $P_{s}<30 \mathrm{~ms}$ pulsars which were more likely to have undergone atleast mild recycling. Details of methodology and notations have been discussed in section 3 which were not provided in our earlier paper (Bagchi \& Ray 2009). Section 3 is further subdivided into $\S 3.1$ for fly-by and $\S 3.2$ for exchange and merger interactions. We report the details of the STARLAB runs used to simulate binary-single stars scattering and provide outputs for some of those runs e.g. cross-sections of different interactions for different stellar parameters, eccentricity - orbital period distribution after exchange/merger interactions etc. Next, in section 4 we have a discussion on the role of ionization in affecting the distribution of pulsar characteristics where we show that ionization at present cannot be responsible for the lack of long period binaries in the observed GCs. In section 5 we discuss our analytical and computational results in relation to the available data and in section 6 we give our conclusions.

\footnotetext{
${ }^{1}$ Information on these pulsars are found from P. Freire's webpage updated August 2008, http://www.naic.edu/ pfreire/GCpsr.html, compiled from radio timing observations by many groups.
} 


\section{Archival data on binary radio pulsars in globular clusters}

We summarize the relevant properties of the host GCs (Webbink 1985) and binary pulsars therein in tables 2 and 3 respectively. In Fig 1, we plot $e$ Vs $P_{\text {orb }}$ for all the binary pulsars with known orbital solutions. Purple $\mathbf{\nabla}$ s are for globular cluster binaries and red $\boldsymbol{\Delta}_{\mathrm{s}}$ are for disk binaries. A logarithmic scale in both eccentricity and orbital periods are chosen, as the enormous range of both variables and the regions occupied by observed pulsars are less obvious in linear scales2. Note that $10 \mathrm{GC}$ binary pulsars have only upper limits to their eccentricities (see table 3). In addition, one galactic disk binary pulsar PSR J1744-3922 $\left(P_{\text {orb }}=0.2\right.$ days $)$ also has only an upper limit to the eccentricity of $<0.001$. Observed binaries in GCs can be categorized in three groups: (I) large eccentricity pulsars $(e \geqslant 0.01)$, 21 pulsars (22 if we include M30B), (II) moderate eccentricity pulsars $\left(0.01>e \geqslant 2 \times 10^{-6}\right)$, 20 pulsars and (III) small eccentricity pulsars $(e \sim 0), 32$ pulsars. In the database, orbital eccentricities of group (III) pulsars have been listed as zero, and in this logarithmic plot we assign them an arbitrarily small value of $e=3 \times 10^{-7}$. Actually for these binaries, it is difficult to measure the eccentricities with present day timing accuracy. This gives a limit to the minimum eccentricity which can be measured as a function of orbital period as given by Phinney (1992) :

$$
\delta t=(e a / 2 c) \sin i\left[\sin \omega+\sin \left(4 \pi t / P_{o r b}-\omega\right)\right]
$$

giving

$$
e_{\text {min }}=(\delta t) /(\operatorname{asin} i / c)=\frac{4 \pi^{2} c}{\sin i\left[G\left(m_{p}+m_{c}\right)^{1 / 3}\right]} \frac{\delta t}{P_{\text {orb }}^{2 / 3}}
$$

where $i$ is the inclination angle of the binary, $\omega$ is the angle between the line of nodes and the line of apsides, $m_{p}$ is the pulsar mass, $m_{c}$ is the companion mass and $\delta t$ is the timing accuracy. We call equation (2) as the "limit of timing sensitivity" and is shown as the solid line in the lower left corner of Fig 1 taking $m_{p}=1.4 M_{\odot}, m_{c}=0.33 M_{\odot}, i=60^{\circ}$, $\delta t=1 \mu \mathrm{sec}$. In addition we plot on the right of Fig 1, dashed lines corresponding to the freeze-out eccentricity - orbital period relation predicted by Phinney (1992) on the basis of the fluctuation dissipation theorem for convective eddies in the erstwhile red giant envelopes surrounding the white-dwarf cores which end up as companions of the neutron stars. This

\footnotetext{
${ }^{2}$ There are three disk binaries with $P_{\text {orb }}>1000$ days such as (i) PSR J0823+0159 with $P_{\text {orb }}=1232.40400$ days, $e=0.0118689$, (ii) PSR J1302-6350 with $P_{\text {or } b}=1236.72404$ days, $e=0.8698869$, (iii) PSR J1638-4725 with $P_{\text {orb }}=1940.9$ days, $e=0.9550000$. These are excluded from our graph as we choose the range of $P_{\text {orb }}$ up to 1000 days because our main concern, globular cluster binaries have always $P_{\text {orb }}<1000$ days.
} 
relation is obtained from the following expression:

$$
\frac{1}{2} \mu \Omega_{b}^{2} a^{2}<e^{2}>=3.4 \times 10^{-5}\left(L^{2} R_{c}^{2} m_{e n v}\right)^{1 / 3}
$$

where $\Omega_{b}=2 \pi / P_{b}$ is the orbital angular rotation frequency, $a$ is the semi-major axis of the binary, $e$ is the orbital eccentricity, $\mathrm{L}$ is the luminosity of the companion, $R_{c}$ is the radius of the companion, $m_{e n v}$ is the envelop mass of the companion, $m_{p}$ is the pulsar mass, $m_{c}$ is the companion mass and $\mu=\left(m_{p} m_{c}\right) /\left(m_{p}+m_{c}\right)$ is the reduced mass of the system. Phinney used $R_{c}=0.125 a$ when the mass transfer ceases and $e$ freezes. Then the above equation reduces to $<e^{2}>^{1 / 2}=C_{1}\left(P_{\text {orb }} / 100\right)$ where $C_{1}$ is a function of $T_{e f f}, \mu$ and $m_{e n v} . P_{o r b}$ is the orbital period in days. Phinney (1992) calculated $C_{1}=1.5 \times 10^{-4}$ using average values of $m_{e n v}$ and $T_{\text {eff }}$ for population I stars $(z=0.04)$ (Refsdal \& Weigert 1970). However for globular clusters, population II stars are more appropriate. So we recalculated $C_{1}$ for population II red giant stars which in general are smaller than population I stars of similar mass and evolutionary stage, and their binary orbital periods at Roche lobe overflow and subsequent mass transfer are shorter than population I red giant binaries (Webbink et al. 1983). We thus obtained $C_{1}=0.86 \times 10^{-4}$. This line is plotted as the lower dashed green line in Fig. 1 .

The galactic field pulsars in the phase space of Fig. 1 1 are also shown here. Observational selection effects may be influencing the distribution seen in this figure for the two sets (Camilo \& Rasio 2005), the most important selection effect being operative towards the left of the diagram, namely, it is more difficult to detect pulsars with larger DM and/or shorter periods, especially millisecond pulsars in very short orbital period and highly eccentric binaries; distance to the pulsars also is an important selection effect, since at large distance only the brightest pulsars can be observed). Nevertheless, it is clear that there is a large abundance of pulsars with long orbital periods and intermediate eccentricity near the eddy "fluctuation dissipation" lines among the galactic disk pulsars which are absent in the GC pulsar population, where the important selection effects are unlikely to play a major role. Other than this, the galactic disk pulsars by and large seem to follow the above three groupings for GC pulsars. The observed preponderance of double neutron star binaries in the field pulsars among the very short orbital period and large eccentricity orbits may be related to selection effects (see above), but we note that there is at least one DNS binary among the GC system: PSR J2127+1105C in M15 in this group (the galactic field DNS binary pulsars are by and large at small distances while M15 and other host GCs are usually far away $\sqrt{3}$.

\footnotetext{
${ }^{3}$ The issue of double neutron star binaries (DNS systems) has been considered by Ivanova et al. (2008). The case for the eccentricity distribution of galactic disk double neutron star (DNS) binaries has been
} 
We have also performed a cluster analysis test using the statistical software " $R$ " 4 for globular cluster binary pulsars. Here we assign random eccentricities of the group III (very low eccentricity) pulsars in such a way that they remain below the "limit of timing sensitivity" line. Performing a K-means test with 3 clusters, we got 3 clusters of sizes 21 (high eccentricity), 18 (medium eccentricity) and 34 (low eccentricity) with the sum of squares from points to the assigned cluster centers as 19.92446, 10.21016 and 13.25632 respectively whereas the squares of inter-cluster distances are as follows $d_{23}^{2}=7.568824, d_{31}^{2}=30.50265$, $d_{12}^{2}=10.85488$. This indicates that the three groupings referred to above are statistically separate cluster of objects.

Formation scenario of binary millisecond pulsars suggests that their eccentricity should be very small, $\sim 10^{-6}-10^{-3}$ (Phinney 1992). But there are many eccentric millisecond pulsars inside globular clusters which are likely to be due to interactions of the binary pulsars with single stars provided the interaction timescale is less than the binary age. We take the maximum age of the binaries in a GC to be the globular cluster ages which are close to $\sim 10^{10}$ years. In subsequent sections, we study different types of stellar interactions and their effect on orbital eccentricities and periods. We include where relevant, the effects of gravitational radiation on these phase space variables.

\section{Orbital parameter changes due to stellar interactions \& gravitational radiation}

Globular clusters contain a large number of low mass X-ray binaries compared to the galactic field; this had led to the suggestion (Fabian et al. 1975) that a binary is formed by tidal capture of a non-compact star by a neutron star in the dense stellar environment of the GC cores. If mass and angular momentum transfer from the companion to the neutron

considered by Ihm, Kalogera \& Belczynski (2006). Since these systems are the result of evolution of massive stars in binary systems, if they formed directly in GCs, they would have formed early in the life of a GC, since the lifetime of a massive star $\left(M \geq 8 M_{\odot}\right)$ is of the order of only $10 \mathrm{Myr}$. Most binaries that have one component forming a neutron star are disrupted in the process of the NS formation, especially those that are in low mass systems, but those that survive tend to have progenitor binaries that were rather tightly bound and subsequent binary-single encounter mostly harden the NS binary. Ivanova et al. (2008) find only 14 DNSs were formed dynamically during the 11 Gyr for all the 70 models of GC population synthesis they consider, most of these would have formed in Ter 5 . The only known case of a DNS in a GC is however that of M15 PSR B2127+1105C. PSR J0514-4002A is a 5-ms pulsar is located in the globular cluster NGC 1851; it belongs to a highly eccentric $(\mathrm{e}=0.888)$ binary system, but the massive companion here may be a white dwarf rather than a neutron star (Freiere, Ransom \& Gupta 2008).

${ }^{4}$ www.R-project.org 
star took place in a stable manner, this could lead to recycled pulsars in binaries or as single millisecond pulsars (e.g. Alpar et al. 1982, Romani et al 1987, Verbunt et al 1987). However large energies may be deposited in tides after tidal capture of a neutron star by a low mass main sequence star in a GC. The resultant structural readjustments of the star in response to the dissipation of the modes could be very significant in stars with either convective or radiative damping zones (Ray, Kembhavi \& Antia 1987; McMillan, McDermott \& Taam 1987) and the companion star can undergo a size "inflation" due to its high tidal luminosity which may exceed that induced by nuclear reactions in the core, by a large factor. Efficiency of viscous dissipation and orbit evolution is crucial to the subsequent evolution of the system as viscosity regulates the growth of oscillations and the degree to which the extended star is bloated and shed. The evolution of the system could lead to either a merger (leading to a Thorne Zytkow object) or a neutron star surrounded by a massive disk comprising of the stellar debris of its erstwhile companion or, in other cases, a low mass X-ray binary or a even detached binary following envelope ejection. Tidal oscillation energy can also be returned to the orbit (i.e. the tide orbit coupling is a dynamical effect) thus affecting the orbit evolution or the extent of dissipative heating in the less dense companion. The evolutionary transition from initial tidal dynamics to any likely final, quiescent binary system is thus regulated by viscosity. Tide orbit coupling can even lead to chaotic evolution of the orbit in some cases (Mardling 1995) but in the presence of dissipation with non-liner damping, the chaotic phase may last only for about 200 yr after which the binary may undergo a periodic circularization and after about 5 Myr finish circularizing (Mardling 1996). Of the stars that do not directly lead to mergers, a roughly equal fraction of the encounters lead to binaries that either become unbound as a result of de-excitation or heating from other stars in the vicinity or they are scattered into orbits with large pericenters (compared to the size of the non-compact star) due to angular momentum transfer from other stars (Kochanek 1992). Thus Kochanek's "intermediate pericenter" encounters can lead to widened orbit (even 100 days orbital period in an eccentric orbit) in tidal encounters involving main sequence stars and neutron stars, which in the standard scenario are attributed to encounters between giants and neutron stars. The complexities of the dynamics of tidal capture and subsequent evolution are manifold and attempts to numerically simulate collisions of neutron stars with red giants have been made with 3D Smoothed Particle Hydrodynamics (SPH) codes (e.g. Rasio \& Shapiro 1991, Davis, Benz \& Hills 1991). We note in this context that Podsiadlowski, Rappaport \& Pfahl (2002) have stated "it is not only premature to rule out tidal capture as a formation scenario for LMXBs, but that LMXBs in globular clusters with well determined orbital periods actually provide observational evidence in its favor". The different formation channels of pulsars in binaries in GCs and the birthrate problems of millisecond pulsar binaries vs LMXBs were considered in (Ray \& Kluzniak 1990; Kulkarni et al 1990). 
Until the early eighties there was no evidence of a substantial population of primordial binaries in any globular clusters, and it was even thought that GCs are significantly deficient in binaries compared to a younger galactic population (Hut et al. 1992). Theoretical modeling of GCs was often started off as if all stars were singles. During the 1980s several observational techniques began to yield a rich population of binary stars in GCs (see Bassa et al. 2008, Yan \& Mateo 1994, Pryor et al. 1989). When the local binary fraction is substantial, the single star - binary interaction can exceed the encounter rate between single stars by a large factor (Sigurdsson \& Phinney 1993). The existence of a significant population of primordial binary population in GCs indicated that three body processes have to be accounted for in any dynamical study of binaries involving compact stars. An encounter between a field star and a binary may lead to a change of state of the latter, e.g.: i) the original binary may undergo a change of eccentricity and orbital period but otherwise remain intact - a "preservation" or a "fly-by" process; ii) a member of the binary may be exchanged with the incoming field star, forming a new binary - an "exchange" process; iii) two of the stars may collide and merge into a single object, and may or may not remain bound to the third star - a "merger" process; iv) three of the stars may collide and merge into a single object - a "triple merger" process; v) all three stars are unbound from an orbit - an "ionization" process. The ionization is always a "prompt" process, whereas the others can be either prompt or "resonant" process. In resonant processes, the stars form a temporarily bound triple system, with a negative total energy but which decays into an escaping star and a binary, typically after 10-100 crossing times.

The value of the binding energy of the binary and the velocity of the incoming star determine the type of interaction the binary will encounter. The critical velocity $v_{c}$ of the incoming star, for which the total energy (kinetic plus potential) of the three body system is zero, can be defined as

$$
v_{c}^{2}=G \frac{m_{1} m_{2}\left(m_{1}+m_{2}+m_{3}\right)}{m_{3}\left(m_{1}+m_{2}\right)} \frac{1}{a_{i n}}
$$

where $m_{1}$ and $m_{2}$ are the masses of the binary members, $m_{3}$ is the mass of the incoming star, $a_{i n}$ is the semi-major axis of the binary and $\mathrm{G}$ is the gravitational constant.

In case of a binary-single star interaction, semi major axis of the final binary $\left(a_{f i n}\right)$ is related to the semi major axis of the initial binary $\left(a_{i n}\right)$ as follows (Sigurdsson \& Phinney 1993):

$$
a_{\text {fin }}=\frac{1}{1-\Delta} \frac{m_{a} m_{b}}{m_{1} m_{2}} a_{i n}
$$

where $m_{1}$ and $m_{2}$ are masses of the members of the initial binary, $m_{a}$ and $m_{b}$ are masses of the members of the final binary. $\Delta$ is the fractional change of binary binding energy i.e. 
$\Delta=\left(E_{i n}-E_{f i n}\right) / E_{i n}$. The binding energies of the initial and final binaries are

$$
E_{\text {in }}=-G \frac{m_{1} m_{2}}{2 a_{i n}}, \quad E_{f i n}=-G \frac{m_{a} m_{b}}{2 a_{f i n}}
$$

For fly-by, $m_{a}=m_{1}, m_{b}=m_{2}$ giving

$$
a_{\text {fin }}=\frac{1}{1-\Delta} a_{i n}
$$

For exchange, $m_{a}=m_{1}, m_{b}=m_{3}$ (star 2 is being replaced by star 3 ) giving

$$
a_{\text {fin }}=\frac{1}{1-\Delta} \frac{m_{1} m_{3}}{m_{1} m_{2}} a_{i n}=\frac{1}{1-\Delta} \frac{m_{3}}{m_{2}} a_{i n}
$$

For merger, $m_{a}=m_{1}, m_{b}=m_{2}+m_{3}$ (star 2 is being merged with star 3 ) giving

$$
a_{\text {fin }}=\frac{1}{1-\Delta} \frac{m_{1}\left(m_{2}+m_{3}\right)}{m_{1} m_{2}} a_{i n}=\frac{1}{1-\Delta} \frac{m_{2}+m_{3}}{m_{2}} a_{i n}
$$

The actual value of $\Delta$ is not known. Putting $\Delta=0$ simplifies equations (5), (7), (8) and (9) giving $a_{f i n}=a_{i n}$ for fly-by interactions. Similar expressions can be derived for other cases e.g. star 1 is being replaced by star 3 or star 1 being merged with star 3 . But in the present work, we always assume star 1 to be the neutron star, so to get binary radio pulsar these latter two processes have to be rejected.

Interactions between binaries and single stars can enhance the eccentricity of the binary orbit and this will be discussed in detail in the subsequent sections. On the other hand, binary pulsars emit gravitational waves which reduce both the size and eccentricity of the orbit leading to mergers on a timescale of $t_{g r}$. Following Peters \& Mathews (1963), $t_{g r}$ can be calculated as follows :

$$
t_{g r}=\left(\frac{1}{e} \frac{d e}{d t}\right)^{-1}
$$

where

$$
\frac{d e}{d t}=-\frac{304}{15} \frac{G^{3} m_{p} m_{c}\left(m_{p}+m_{c}\right)}{c^{5} a_{p}^{4}} g(e)
$$

and

$$
g(e)=\left(1-e^{2}\right)^{-5 / 2} e\left(1+\frac{121}{304} e^{2}\right)
$$

which is almost same as the expression of $t_{g r}$ calculated from $d a / d t$. 


$$
\begin{gathered}
t_{g r}=\left(\frac{1}{a} \frac{d a}{d t}\right)^{-1} \\
\frac{d a}{d t}=-\frac{64}{5} \frac{G^{3} m_{p} m_{c}\left(m_{p}+m_{c}\right)}{c^{5} a_{p}^{3}} f(e)
\end{gathered}
$$

and

$$
f(e)=\left(1-e^{2}\right)^{-7 / 2}\left(1+\frac{73}{24} e^{2}+\frac{37}{96} e^{4}\right)
$$

In this work, we use eq. (10) to calculate $t_{g r}$.

\subsection{Fly-by interactions}

Table 3 shows that most of the GC pulsars are millisecond pulsars. Theoretically one expects that spun-up, millisecond pulsars in binary systems formed from mass and angular momentum transfer due to Roche lobe overflow and the resultant tidal effects should appear in low eccentricity orbits $e \sim 10^{-6}-10^{-3}$, (Phinney 1992). Since many highly eccentric binary millisecond pulsars are found in globular clusters, this indicates that stellar interactions are important for inducing higher eccentricities. Rasio and Heg-

gie (Rasio \& Heggie 1995; Heggie \& Rasio 1996) studied the change of orbital eccentricity of an initially circular binary following a distant encounter with a third star in a parabolic orbit. They used secular perturbation theory, i.e. averaging over the orbital motion of the binary for sufficiently large values of the pericenter distance $r_{p}$ where the encounter is quasi-adiabatic and used non secular perturbation theory for smaller values of $r_{p}$ where the encounter is non-adiabatic. In the first case $\delta e$ varies as a power law with $r_{p} / a_{i n}$ and in the second case $\delta e$ varies exponentially with $r_{p} / a_{i n}$. The power law dominates for $e<0.01$ and the exponential dominates for $e \gtrsim 0.01$. They used the relation

$$
b^{2}=r_{p}^{2}+2 G r_{p}\left(m_{1}+m_{2}+m_{3}\right) / v^{2} \simeq 2 G r_{p}\left(m_{1}+m_{2}+m_{3}\right) / v^{2}
$$

to estimate the cross-sections $\left(\sigma=\pi b^{2}\right)$ for eccentricity changes and then from $\sigma$, obtained the time-scales for eccentricity changes as $t=1 /($ rate $)=1 /<n \sigma v>$ where $n$ is the number density. The timescales for these processes are (Rasio \& Heggie 1995):

$$
\begin{array}{rr}
t_{f l y}=4 \times 10^{11} n_{4}^{-1} v_{10} P_{\text {orb }}^{-2 / 3} e^{2 / 5} & (\mathrm{e} \lesssim 0.01) \\
t_{f l y}=2 \times 10^{11} n_{4}^{-1} v_{10} P_{\text {orb }}^{-2 / 3}[-\ln (e / 4)]^{-2 / 3} & (\mathrm{e} \gtrsim 0.01)
\end{array}
$$


where $n_{4}$ is the number density $(n)$ of single stars in units of $10^{4} \mathrm{pc}^{-3}$ and $v_{10}$ is the velocity dispersion $(v)$ in units of $10 \mathrm{~km} / \mathrm{sec}$ in GCs, $P_{\text {orb }}$ is the orbital period in days giving $t_{f l y}$ in years. The values of $v_{10} / n_{4}$ are different for different GCs (table 21) and we grouped them into six different groups according to their values of $v_{10} / n_{4}$ and calculated $t_{\text {fly }}$ for mean values of $v_{10} / n_{4}$ for each group. Cluster binary pulsars in the $e-P_{\text {orb }}$ plane with contours of $t_{f l y}=10^{10} \mathrm{yrs}$ (solid lines) and $t_{f l y}=10^{8} \mathrm{yrs}$ (dashed lines) for each group are shown in Fig 2. Contours of $t_{g r}=10^{10}$ yrs (solid lines) and $t_{g r}=10^{8}$ yrs (dashed lines) for binaries with $m_{p}=1.4 M_{\odot}$ and $m_{c}=0.35 M_{\odot}$ or $0.16 M_{\odot}$ are also shown. Pulsars with projected positions inside the cluster core are marked with + , those outside the cluster core are marked with $\times$ and the pulsars with unknown position offsets are marked with $\diamond$. Solid red line corresponding to $t_{f l y}=10^{10}$ years for $v_{10} / n_{4}=0.0024$ is outside the range of axes used in our plots (away from the upper left corner). Individual pulsars are marked with the same colors as the $v_{10} / n_{4}$ values of the host GCs. If a pulsar is located on the upper left half of the corresponding $t_{f l y}=10^{10}$ years line i.e. in the region where $t_{f l y}>10^{10}$ years, then its eccentricity cannot be due to fly-by interactions. There are two such binaries, one is PSR B1718-195 (in NGC 6342, in group 4) and the other is PSR B 1639+36B (in M13, in group 6 ). But the first one is a normal pulsar with $P_{s}=1 \mathrm{sec}$ and as it is not spun up and may not have been subject to a great deal of tidal forces, it can have escaped circularization. On the other hand, the second pulsar is a millisecond pulsar with $P_{s}=3.528 \mathrm{~ms}$, so it should have been in a circular orbit if it has not had the time to go through any significant kind of stellar interactions. Therefore it is of interest to investigate if it is a result of an exchange or merger interaction. Moreover, some of the eccentric pulsars which seem to be explainable by fly-by interactions lie outside of globular cluster cores (which have less stellar density than in the core, and therefore less efficient fly-by scattering). For a few others positional offsets are not known. Even if a pulsar appears to be inside the cluster core in the projected image, it can still be outside the cluster core in three dimensional space. Globular cluster models show that values of both $v_{10}$ and $n_{4}$ fall outwards from cluster center and as the fall of $n_{4}$ is much more rapid, the value of $v_{10} / n_{4}$ is higher outside the cluster core. As we have already seen (different panels in Fig. (2) that an increase in the value of $v_{10} / n_{4}$ shifts the contour of $t_{f l y}=10^{10}$ years rightwards, this will make the pulsar fall in the region where $t_{f l y}>10^{10}$ years excluding the possibility of fly-by interaction as the cause of its eccentricity. In those cases also, we need to think about exchange and/or merger interactions. Another aspect of the pulsar population in these diagrams is that the long orbital period pulsars are found predominantly in low density (and high velocity dispersion) GCs, a feature that has been noticed before (Camilo \& Rasio 2005). Camilo \& Rasio (2005) also point out that while the

\footnotetext{
${ }^{5}$ Note however that its cluster association is sometimes doubted, - see (Camilo \& Rasio 2005), as it has a large offset from the GC center.
} 
LMXBs are predominantly found in very dense GCs, the radio pulsars tend to be found more evenly distributed among low and high density clusters, as also seen in the great deal of variation of the $v_{10} / n_{4}$ parameter in the various plots of Fig. 2 .

We have seen that there are many zero eccentricity pulsars which lie in the region where $t_{f l y}<10^{10}$ years. So it is of interest to understand why they still appear in circular orbits despite the possibility of fly-by interactions. It is possible however that, firstly the binaries may be very young so that they have not had enough time to interact with single stars. Secondly, they may in fact lie outside cluster cores with a higher $v_{10} / n_{4}$ even though in projection many of them may appear to be inside the cores so that the timescale for fly-by is actually $t_{f l y}>10^{10}$ years.

Positions of cluster binaries with respect to the cluster center in the projected plane is given in Table 3. There are a number of pulsars for which position offsets are not known. Also the binaries which appear to be inside the cluster core in the projected plane can actually lie outside the core. The observed values of spin period derivatives $\left(\dot{P}_{s}\right)$ of the globular cluster pulsars can give some indication of the pulsar position and its environment since negative or positive $\dot{P}_{s}$ indicate positions in the back half or in the front half of the cluster respectively (Phinney 1992); however, the actual position cannot be determined with the knowledge of $\dot{P}_{s}$ only. DM measure could also be a tool to measure pulsar positions with respect to cluster center if DM variations were dominated by intra-cluster gas (Ransom 2006). However, it has been shown that for Terzan 5, DM is mainly due to ISM (Ransom2006), so DM values alone can not help to determine pulsar positions with respect to the center for this cluster.

\subsection{Exchange and merger interactions}

Since exchange and merger interactions have not been amenable to analytic treatment, we performed numerical scattering experiments using STARLAB codes 6 . We used the STARLAB task "sigma3" which gives the scaled cross section $X$ for different types of interactions, e.g. fly-by, exchange, merger and ionization (both resonant and non-resonant). The inputs given are : velocity $(v)$ of the incoming star (with mass and radius $m_{3}, R_{3}$ ) and the masses, the radii and the semi major axis of the initial binary $\left(m_{1}, R_{1} ; m_{2}, R_{2} ; a_{i n}\right)$. $X$ is defined as :

$$
X=\frac{\sigma}{\pi a_{i n}^{2}}\left(\frac{v}{v_{c}}\right)^{2}
$$

where $\sigma$ is the cross section and $v_{c}$ is the critical velocity as defined earlier (eq. 4).

\footnotetext{
${ }^{6}$ www.ids.ias.edu/ starlab/
} 
We select the range of $a_{i n}$ as $0.001-8.0 A U$ so that for all sets of stellar parameters, the initial orbital periods are well within the range of observed values of orbital periods 0.01 - 1000 days. But depending upon the stellar parameters, one or more values of $a_{i n}$ are automatically rejected by STARLAB whenever $a_{i n}$ is too low (giving "contact binaries"). We divide the range of $a_{i n}$ in 35 bins $\left(N_{b i n}\right)$ equal in logarithmic scales to invoke "sigma3". For each value of $a_{i n}$, we take the maximum trial density $\left(n_{\text {trial }}\right)$ as 5000 which is uniformly distributed in the impact parameter $(\rho)$ over the range $0 \leq \rho \leq \rho_{0}$ where $\rho_{0}$ simply corresponds to a periastron separation of $2 a$. The impact parameter range is then systematically expanded to cover successive annuli of outer radii $\rho_{i}=2^{i / 2} \rho_{0}$ with $n_{\text {trial }}$ trials each until no interesting interaction take place in the outermost zone (McMillan 1996). Thus the total number of trials become significantly large. As an example, for the parameters $m_{1}=1.4 M_{\odot}, R_{1}=10 \mathrm{~km} ; m_{2}=0.16 M_{\odot}, R_{2}=0.16 R_{\odot} ; m_{3}=$ $0.33 M_{\odot}, R_{3}=R_{\odot} ; a_{i n}=0.2 \mathrm{AU} ; v=11.76 \mathrm{~km} / \mathrm{sec}$ we had a sample total of 15570 scatterings. Out of these 10960 were fly-bys, 3722 exchanges, 982 two mergers and 6 threemergers. The CPU time needed to perform this particular set of interaction was 1171.23 secs 7 . Though widely different values of $v$ were chosen $(13.15 \mathrm{~km} / \mathrm{sec}, 11.76 \mathrm{~km} / \mathrm{sec}, 7.79$ $\mathrm{km} / \mathrm{sec}$ and $3.29 \mathrm{~km} / \mathrm{sec}$ ) covering the entire range of $v$ in $26 \mathrm{GCs}$, we concentrated mainly on the runs for $v=11.76 \mathrm{~km} / \mathrm{sec}$ corresponding to the velocity dispersion relevant to Terzan 5 , the host of the largest number of known binary radio pulsars. Different parameters for STARLAB runs performed for this value of $v$ are given in Table 1 ,

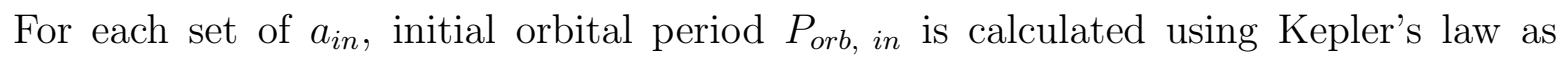
stellar parameters are known. STARLAB outputs contain X corresponding to each $a_{\text {in }}$ i.e. corresponding to each $P_{\text {orb }}$ in for all types of interactions. In Fig. 3 we plot the variation of $X$ for exchange $(+)$, merger $(\boldsymbol{\Delta})$ and ionization (o, whenever significant) with $P_{\text {orb }, \text { in }}$ and check the dependencies on different parameters - (i) In the first figure, we set $m_{1}=1.4 M_{\odot}, m_{2}=0.16 M_{\odot}, m_{3}=0.33 M_{\odot}$, and take two values of $v$ e.g. 13.16 $\mathrm{km} / \mathrm{sec}$ (red) and $3.27 \mathrm{~km} / \mathrm{sec}$ (green) which are the highest and the lowest values (see Table 2). A change in the value of $v$ does not change the value of $X$ much when the other parameters are kept fixed. (ii) In the second figure, we set $m_{1}=1.4 M_{\odot}, m_{3}=0.33 M_{\odot}$, $v=11.76 \mathrm{~km} / \mathrm{sec}$, and take different values of $m_{2}$ e.g. $0.40 M_{\odot}$ (red), $0.16 M_{\odot}$ (green) and $0.024 M_{\odot}$ (blue). Both $X_{\text {exchange }}$ and $X_{\text {merger }}$ increases with decrease of $m_{2}$. Ionization starts for $m_{2}=0.024 M_{\odot}$ at $P_{\text {orb }, \text { in }} \approx 200$ days. (iii) In the third figure, we set $m_{1}=1.4 M_{\odot}, m_{2}=0.16 M_{\odot}, v=11.76 \mathrm{~km} / \mathrm{sec}$, and take different values of $m_{3}$ e.g. $0.33 M_{\odot}$ (red), $0.80 M_{\odot}$ (green). Both $X_{\text {exchange }}$ and $X_{\text {merger }}$ increases with increase of $m_{3}$.

\footnotetext{
${ }^{7}$ The simulations were performed on a HP Proliant BL465C computer with Dual-Core $2.6 \mathrm{GHz}$ AMD Opteron Processor 2218 with 16 GB RAM.
} 
(iv) In the fourth figure, we set $m_{1}=1.4 M_{\odot}, m_{2}=0.16 M_{\odot}, m_{3}=0.80 M_{\odot}, v=11.76$ $\mathrm{km} / \mathrm{sec}$ and take the $3^{\text {rd }}$ star to be either a MS (red) or a WD (green). For WD, $X_{\text {exchange }}$ is higher but $X_{\text {merger }}$ is lower.

From $X$, one can calculate the cross-section ( $\sigma$ see eq. 17) and the interaction time scale $t$ as $t=1 / n \sigma v$ where $n$ is the number density of single stars. STARLAB also gives the properties of final states, i.e. eccentricities and semi major axes of the final binaries for each set of inputs $\left(m_{1}, R_{1} ; m_{2}, R_{2} ; m_{3}, R_{3} ; a_{i n}\right)$. So for each value of $P_{\text {orb }}$, in , we get a number of values of $P_{\text {orb }}$, fin and $e_{\text {fin }}$. For each value of $P_{\text {orb }}$, in, we calculated 25 percentile, median and 75 percentile values of $P_{\text {orb }}$, fin as well as the values from analytic expressions given in eq. 8 and 9 putting $\Delta=0$. As all these values are very close to each other, we use the $P_{\text {orb }, \text { in }}-P_{\text {orb, fin }}$ relation corresponding to $\Delta=0$ throughout. As an example, in Fig. 4, we have plotted $P_{\text {orb }}$, in $\mathrm{Vs} P_{\text {orb }}$, fin as reported by STARLAB (scatter plots) for both merger and exchange with $m_{1}=1.4 M_{\odot}, m_{2}=0.16 M_{\odot}, m_{3}=0.33 M_{\odot}, v=11.76$ $\mathrm{km} / \mathrm{sec}$. The line for 25 percentile, median, 75 percentile along with the analytic relation of $P_{\text {orb }, \text { in }}-P_{\text {orb, fin }}$ with $\Delta=0$ (eq. 5) are shown in the same plot.

In Fig. 5, we plot $P_{\text {orb,in }}$ of the initial binary (comprising of stars $m_{1}$ and $m_{2}$ ) along the top x-axis and $P_{\text {orb,fin }}$ along the bottom x-axis. Purple points in the left panels are for exchange interactions while the green points in the right panel are for the merger interactions. The left $\mathrm{y}$ axis gives the final eccentricities while the right $\mathrm{y}$ axis gives the time scales of the interactions. Interaction time scales are plotted with black $+\mathrm{s}$. The vertical orange lines form the boundaries of the allowed orbital period regions where interaction time scales are less than $10^{10}$ years. It is clear from the scatter plots (Fig. 5) that the final binaries will most probably have $e>0.1$ if they undergo either exchange or merger events. The observed pulsars with $e>0.1$ in Terzan 5 can be found in Table 3 where the companion masses $\left(m_{c}\right)$ are for inclination angle $i=60^{\circ}$ and are also shown in the scatter plots (red in color, named in few plots). In Fig [5, $m_{1}=1.4 M_{\odot}, m_{3}=0.50 M_{\odot}$ and we vary $m_{2}$ from $0.024 M_{\odot}$ to $0.16 M_{\odot}$ and $0.40 M_{\odot}$. In Table1, we summarize the stellar parameters for the different sets of STARLAB runs.

If an observed eccentric binary pulsar lies in the region where time scale for a particular interaction is greater than $10^{10}$ years, then that interaction can not be responsible for its eccentricity. On the other hand, an exchange interaction to be the origin of the eccentricity of a particular pulsar, we should have $m_{c} \approx m_{3}$ and for merger $m_{c} \approx m_{2}+m_{3}$. Considering these two facts, we can surmise that PSR I may have resulted from exchange interaction $m_{2}$ is either $0.16 M_{\odot}$ or $0.40 M_{\odot}$, PSR Q may have resulted from merger interaction when $m_{2}$ is $0.16 M_{\odot}$, PSR $\mathrm{U}$ can result from exchange interaction when $m_{2}$ is either $0.16 M_{\odot}$ or $0.40 M_{\odot}$ or from merger interaction when $m_{2}$ is $0.16 M_{\odot}$, PSR Z can result from merger 
interaction when $m_{2}$ is either $0.16 M_{\odot} 0.40 M_{\odot} 8$. These conditions on stellar masses are mainly indicative and need not be satisfied very accurately because: 1) a slightly different choice of pulsar masses can give the same or similar output of the simulation and 2) the companion masses are not known exactly in most cases; the masses are obtained from the mass function in terms of $m_{c} \sin i$ and with the assumption that the orbital inclination angle is $60^{\circ}$ (Table 3).

\section{Ionization}

STARLAB runs show that ionization starts only at high values of $P_{\text {orb }}$ and a lower value of $m_{2}$ or a higher value of $m_{3}$ can facilitate ionization for obvious reasons of available kinetic and binding energies. As an example, keeping $m_{1}=1.4 M_{\odot}$ and $m_{3}=0.33 M_{\odot}$ fixed, the minimum value of $P_{\text {orb }}$ increases from $\sim 200$ days to $\sim 3000$ days when we increase $m_{2}$ from $0.024 M_{\odot}$ to $0.16 M_{\odot}$. For the case of $m_{2}=0.40 M_{\odot}$, ionization starts after 10000 days. In Fig. 6, we show the variation of the cross section $\sigma$ with $P_{\text {orb }}$ for different interaction with $m_{1}=1.4 M_{\odot}, m_{2}=0.024 M_{\odot}, m_{3}=0.33 M_{\odot}$; this is the only case where ionization starts in the orbital period range of the observed globular cluster binaries. This happens because the condition of ionization e.g. $v / v_{c}>1$ is satisfied (where $v_{c}$ is the critical velocity giving three unbound stars at zero energy defined in Eq. 4). We have verified that STARLAB outputs also match with the analytical expression of scaled cross section $X$ given by (Spitzer 1987)

$$
X=\frac{\sigma}{\pi a_{i n}^{2}}\left(\frac{v}{v_{c}}\right)^{2}=\frac{40}{3} \frac{m_{3}^{3}}{m_{1} m_{2}\left(m_{1}+m_{2}+m_{3}\right)}
$$

There are three disk pulsar with $P_{\text {orb }}>1000$ days as J0823+0159 $\left(P_{\text {orb }}=1232.40\right.$ days, $\left.M_{c}=0.23 M_{\odot}\right)$, J1302-6350 $\left(P_{\text {orb }}=1236.72\right.$ days, $\left.M_{c}=4.14 M_{\odot}\right)$ and $\mathrm{J} 1638-4725$ $\left(P_{\text {orb }}=1940.9\right.$ days, $\left.M_{c}=5.84 M_{\odot}\right)$. But such high period pulsars have not been observed in globular clusters. If the evolution of neutron star binaries are nearly the same in globular

\footnotetext{
${ }^{8}$ Comparing Fig 5 of the present manuscript with the Fig 2 of Bagchi \& Ray (2009), it is clear that with a change of the value of $m_{3}$ from $0.33 M_{\odot}$ to $0.50 M_{\odot}$, the outcomes of the simulations i.e. the appearance of the scatter plots does not change much in comparison to its change with the change in the value of $m_{2}$ (for fixed $m_{3}$ ). For the sake of completeness, we wish to remind the readers that if $m_{3}$ is $0.33 M_{\odot}$, then PSR I may have resulted from exchange interaction when $m_{2}$ is either $0.16 M_{\odot}$ or $0.40 M_{\odot}$, or merger interaction when $m_{2}$ is $0.16 M_{\odot}$, PSR $\mathrm{J}$ could have resulted from exchange interaction when $m_{2}$ is either $0.16 M_{\odot}$ or $0.40 M_{\odot}$, PSR Q may have resulted from merger when $m_{2}$ is $0.40 M_{\odot}$, PSR U can result from merger interaction when $m_{2}$ is $0.40 M_{\odot}$, PSR X can result from exchange interaction when $m_{2}$ is either $0.16 M_{\odot}$ or $0.40 M_{\odot}$, PSR Z can result from merger interaction when $m_{2}$ is $0.40 M_{\odot}$.
} 
clusters as that in the galactic disk, there should have been similar long period binaries with comparatively massive companions for which ionization cannot be very effective as seen from our calculations. It is possible that such systems have not yet been observed because of observational selection effects, as long period searches require correspondingly long time baselines. Perhaps future pulsar searches with better sensitivity and longer time baselines may reveal such binaries.

\section{Discussions}

Three globular cluster binary pulsars with $0.01<e<0.1$ and $60<P_{\text {orb }}<256 \mathrm{~d}$ (see Fig 1) in M53, M3(D), and Ter 5(E) have companions of mass in the range $m_{c}=$ $0.21-0.35 \mathrm{M}_{\odot}\left(i=60^{\circ}\right)$. These are possibly white dwarf cores of red giant companions that overflowed Roche lobe (Webbink et al. 1983). Such binaries would normally have the "relic" eccentricities $10^{-4}$ (green dashed lines). The above binary pulsars with their presently mildly high eccentricities, may have undergone fly-by encounters with field stars, rather than exchange or merger interactions, which would have produced very high eccentricities $e>0.1$. Ter $5 \mathrm{E}$ lies outside the core where $v_{10} / n_{4}$ is higher than the central value, but even then, it might have been be low enough to allow a strong fly-by interaction. It could also have been ejected out of the high density core after a strong interaction.

Another set of three globular cluster millisecond pulsars have $0.01<e<0.1,2<P_{\text {orb }}<$ $10 \mathrm{~d}$; Ter $5(\mathrm{~W}), 47$ Tuc $(\mathrm{H})$ and NGC6440 (F). All these clusters have low values of $v_{10} / n_{4}$ (Table 2), and so fly-by encounters in these clusters would be efficient and could generate these eccentricities in GCs, even if their progenitor binaries had shorter orbital periods and had sub-giant companions of the NSs like the galactic equivalents of the red triangles of Fig 1. Alternately, these binaries could also have been formed by fly-by interactions from a now less abundant longer period $2<P_{\text {orb }}<10$ day cluster of "intermediate eccentricity" binaries to the right of the pulsars (seen among the purple triangles in the middle of Fig. 1).

The "intermediate eccentricity" binary pulsars (group II : $0.01>e \geq 2 \times 10^{-6}$ ), themselves could have been generated by fly-by encounters with low (or "zero") eccentricity progenitor pulsars below the line of "timing sensitivity limit" (group III pulsars). A great majority of group II pulsars are millisecond pulsars occurring in GCs with high probability of fly-by encounters. But if some of the shorter $P_{\text {orb }}(\sim 0.2$ day $)$ pulsars have indeed been kicked-up to even higher eccentricities in the past, their eccentricity would be reduced by gravitational radiation again. The progenitor group III pulsars, themselves occur in regions of favorable fly-by encounters inducing higher eccentricities. These nearly circular binaries have their $P_{\text {orb }}$ in the range of $0.106-4.0$ days. They can not be results of exchange or 
merger as these processes produce high eccentricities. Camilo \& Rasio (2005) discussed the dynamical formation of ultra-compact binaries involving intermediate mass main sequence stars in the early life of the GC as the origin of the group III pulsars. These companions must have been massive enough (beyond the present day cluster turn-off mass of $0.8 M_{\odot}$ ) so that the initial mass transfer became dynamically unstable, leading to common envelope evolution and subsequent orbital decay and circularization. Alternately, the present day red giant and NS collisions lead to a prompt disruption of the red giant envelope and the system ends up as eccentric NS-WD binary (Rasio \& Shapiro 1991). These binaries could decay to the group III pulsars by gravitational radiation if they had short $P_{\text {orb }} \leq 0.2 \mathrm{~d}$.

Despite the circumstance that most radio pulsars in GCs can be explained by fly-by and/or exchange interactions, there exists a pulsar like the PSR B1639+36B (i.e. M13B) which is a recycled pulsar and in a region of the phase space that is difficult to reach with either fly-by or exchange mechanisms. Systems like this could have undergone eccentricity pumping of the inner orbit due to the presence of a third star in a wide outer orbit, i.e. in a hierarchical triplet like the PSR B1620-26 in M4 system (Sigurdsson 1993; Rasio et al. 1995; Thorsett et al. 1999; Ford et al. 2000). Rasio et al. (1995) showed that only one such triple system can form in a globular cluster like M4, but in clusters with higher values of $n_{4}$ and/or binary fraction the number can be a few, though at present PSR B1620-26 is the only known triple system in globular clusters. A similar mechanism has been suggested recently (Champion et al. 2008) for a galactic disk binary pulsar PSR J1903+0327, a radio pulsar with a rotational period of 2.15 milliseconds in a highly eccentric $(e=0.44)$ 95-day orbit around a solar mass companion star. The formation scenarios for such an unusual pulsar both in and out of GCs, and in particular the observational consequences for further radio timing of PSR J1903+0327 in terms of eccentricity pumping taking account of relativistic precession of the inner orbit periastron, has been investigated (Gopakumar et al. 2009).

\section{Conclusion}

We find that the presently observed orbital eccentricity and period data of GC binary pulsars are largely explained by numerical scattering experiments on stellar interaction scenarios of fly-bys and exchanges with field stars. Binaries with $e>0.1$ are most probably the result of exchange or merger events whereas binaries with $0.01>e>0.00001$ are products of fly-by of single stars. A number of wide orbit intermediate eccentricity pulsars seen in the galactic field are absent in the GC sample because they have been kicked up to relatively high eccentricities by passing stars in the dense stellar environments in GCs. In some GCs such as Ter 5, the stellar densities are so high, and the velocity dispersion so modest that the 
interaction timescale for exchange and fly-by interactions is relatively short. In such GCs a typical binary system may undergo multiple interactions. If the original binary contains a spun-up millisecond pulsar in a relatively "soft" binary, then the exchange interaction may produce a single millisecond pulsar in the cluster. This may explain the higher incidence of single ms PSRs in GCs compared to that in the galactic disk (Camilo \& Rasio 2005). Terzan 5, for example, contains 16 single ms PSRs out of a total of 33 pulsars. In addition, exchange interactions, as we have seen, can lead to highly eccentric orbits and the system can be ejected from the cluster core. If the last encounter took place not too long ago, the system can be at a relatively large offset from the cluster core, albeit being still spatially co-located with the GC.

It is however clear that there are systems in GCs whose orbital characteristics cannot be explained by purely episodic interactions; an example of this kind is PSR B1620-26, which is a hierarchical triplet, whose pulsar orbital eccentricity is affected by the presence of a third star in a loosely bound system. There are possibly other examples of a similar kind where there is eccentricity pumping from the long term presence of companions in a triplet system.

We have also considered the effects of collision induced ionization on the present day distribution of orbital parameters of radio pulsars in GCs. In the galactic disk we find that there exist several pulsars with $P_{\text {orb }}>1200 d$ (although some of them have more massive companions than the pulsar binaries in the GCs), while the similar wide orbit binaries are missing from the GCs. While it is tempting to speculate that these are missing from the present day GCs because they have been ionized in the past, we find that the ionization probability becomes substantial only in a restricted domain of masses of the companion and incoming stars, and that too for essentially periods greater than $P_{\text {orb }}>1000 d$. Binary pulsars with very low companion masses can be ionized easily. On the other hand the apparent lack of the corresponding examples of galactic disk long orbital period binaries surviving due to their massive companions in GCs could also be due to observational selection effects. While ionization interaction can explain lack of binary pulsars with $P_{\text {orb }}>1000 d$ in globular clusters, this process is currently not important, but could have played a role in the past for very long period binaries. On the other hand there are somewhat wide binaries in the present day GCs with moderately high eccentricities (e.g. $0.01<e<0.1$ and $60<P_{\text {orb }}<256 \mathrm{~d}$ ) which could have arisen out of fly-by exchanges from progenitor binaries with "relic" eccentricities $e \sim 10^{-4}$. Many galactic disk binary pulsars are seen in the $e-P_{\text {orb }}$ plane predicted by Phinney due to the fluctuation dissipation of convective eddies and the resultant orbital eccentricities that are induced. These pulsars are missing from the GC sample. There is no reason not to expect these systems to form in the GCs (although due to the lower metallicity of the stellar companions in GCs, they are expected to lead to somewhat less wide systems (Webbink et al. 1983)). This can be explained by the 
substantial probability of them being knocked out of their original phase space due to flyby interaction in GCs.

We thank Roger Blandford, Avinash Deshpande, Shri Kulkarni, Douglas Heggie, Michael Kramer, Ben Stappers, Duncan Lorimer, Paulo Freire, Maura McLaughlin, Andrew Fruchter, Jason Kalirai, Scott Ransom, Avi Loeb, Josh Grindlay, Daniel Fabrycky, Saul Rappaport, Victoria Kaspi, Patrick Lazarus, Fernando Camilo, Rino Bandiera, Sandro Mereghetti, Pierre Pizzochero and Masimo Turatto for discussions and Sayan Chakraborti for comments on the manuscript. We thank the STARLAB development group for the software and the ATNF pulsar group and Paulo Freire for pulsar data bases. We thank the participants and organizers of the $2^{\text {nd }}$ IIA-PennState Astrostatistics School (Kavalur, 2008), in particular Jogesh Babu, Sabyasachi Chatterjee and Prajval Shastri for discussions. This manuscript was completed at Raman Research Institute and we thank its Director, Ravi Subrahmanyan and its staff for

their hospitality on multiple occasions. This research is a part of 11 th plan project 11P-409 at TIFR.

\section{REFERENCES}

Alpar, M. A., Cheng, A. F., Ruderman, M. A. \& Shaham, J., 1982, Nature, 300, 728.

Bagchi, M. and Ray, A., 2009, ApJ, 693, L91.

Bassa, C. G., van Kerkwijk, M. H., Koester, D., Verbunt, F., 2006, A \& A, 456, 295.

Bassa, C. G., Pooley, D., Verbunt, F., Homer, L., Anderson, S. F., Lewin, W. H. G., 2008, A \& A, 488, 921.

Camilo, F. \& Rasio, F. A., 2005, Astron Soc Pac Conf Ser, 328, 147.

Champion, D. J., et al., 2008, Science, 320, 1309.

Davies, M.B., Benz, W., Hills, J. G., 1991, ApJ, 381, 449.

Fabian, A., Pringle, J. \& Rees, M., 1975, MNRAS, 172, 15.

Ford, E. B., Joshi, K. J., Rasio, F. A., Zbarsky, B., 2000, ApJ, 528, 336.

Freire, P. C., Camilo, F., Kramer, M., Lorimer, D. R., Lyne, A. G., Manchester, R. N., D'Amico, N; 2003, MNRAS, 340,1359. 
Freire, P. C. C., Hessels, J. W. T., Nice, D. J., Ransom, S. M., Lorimer, D. R., \& Stairs, I. H. 2005, ApJ, 621, 959.

Freire, P. C. C., Ransom, S. M.; Gupta, Y., 2007, ApJ, 662,1177.

Freiere, P., Ransom, S. M., \& Gupta, Y., 2008, AIP Conference Proceedings, 983, 491.

Freire, P., et al. 2008, ApJ, 675, 670.

Freire, P.C. C., Wolszczan, A., van den Berg, M., Hessels, J.W. T., 2008, ApJ, 679, 1433.

Gopakumar, A., Bagchi, M., Ray, A., 2009, submitted.

Heggie, D. C. \& Rasio, F. A., 1996, MNRAS, 282, 1064.

Hut, P., et al., 1992, PASP, 104, 981.

Hut, P., Shara, M. M.; Aarseth, S. J.; et al., 2003, New Astron, 8, 337.

Ihm, C.M., Kalogera, V., \& Belczynski, K., 2006, ApJ, 652, 540.

Ivanova, N., Heinke, C. O., Rasio, F., Belczynski, K., Fregeau, J. M., 2008, MNRAS, 386, 553.

Jacoby, B. A., Cameron, P. B., Jenet, F. A., Anderson, S. B., Murty, R. N., Kulkarni, S. R., 2006, ApJ, 644, L113.

Kochanek, C., 1992, ApJ, 385, 640.

Kulkarni, S. R., Narayan, R. \& Romani, R. W., 1990, ApJ, 356, 174.

Lyne, A. G. et al., 1987, Nature, 328, 399.

Mardling, R., 1995, ApJ, 450, 722.

Mardling, R., 1996, Astron Soc Pac Conf Ser, 90, 399.

McMillan, S. L. W., 1996, ASPC, 90, 413.

McMillan, S.L.W., McDermott, P. N. \& Taam, R., 1987, ApJ, 318, 261.

Peters, P. C. \& Mathews, J., 1963, Phys. Rev., 131, 435.

Phinney, E. S., 1992, Phil. Trans. R. Soc. Lond., 341, 39.

Podsiadlowski, P., Rappaport, S. \& Pfahl, E. D., 2002, ApJ, 565, 1107. 
Prince, T. A. et al, 1991, Astrophys. J., 374, L41.

Pryor, C., et al., 1989, in Dynamics of Dense Stellar Systems, D. Merrit, Eds. (Cambridge Univ Press, Cambridge, UK, 1989), p. 175.

Ransom, S., 2006, ASP Conference Series, 365, 265.

Rasio, F. A. \& Heggie, D. C., 1995, ApJ, 445, L133.

Rasio, F.A., McMillan, S., Hut, P., 1995, ApJ, 438, L33.

Rasio, F. A. \& Shapiro, S. L., 1991, ApJ, 377, 559.

Ray, A., Kembhavi, A. K., \& Antia, H. M., 1987, A \& A, 184, 164.

Ray, A., \& Kluzniak, W., 1990, Nature, 344, 415.

Refsdal, S., Weigert, A., 1970, A \& A, 6, 426.

Romani, R. W., Kulkarni, S. R. \& Blandford, R., 1987, Nature, 329, 309.

Sigurdsson, 1993, ApJ, 415, L43.

Sigurdsson, S. \& Phinney, E. S., 1993, ApJ, 415, 631.

Verbunt, F., van den Heuvel, E.P.J., van Paradijs, J. \& Rappaport S. A., 1987, Nature, 329, 312.

Spitzer, L., "Dynamical Evolution of globular clusters", Princeton University Press (1987).

Thorsett, S. E., Arzoumanian, Z., Camilo, F., \& Lyne, A. G., 1999, Astrophys. J., 523, 763.

Webbink, R. F, in Dynamics of star clusters Proceedings of the IAU Symposium, Princeton, Dordrecht, D. Reidel Publishing Co.p. 541-577 (1985).

Webbink, R. F., Rappaport, S. \& Savonije, G. J., 1987, ApJ, 270, 678.

Yan, L. \& Mateo, M., 1994, AJ, 108, 1810. 


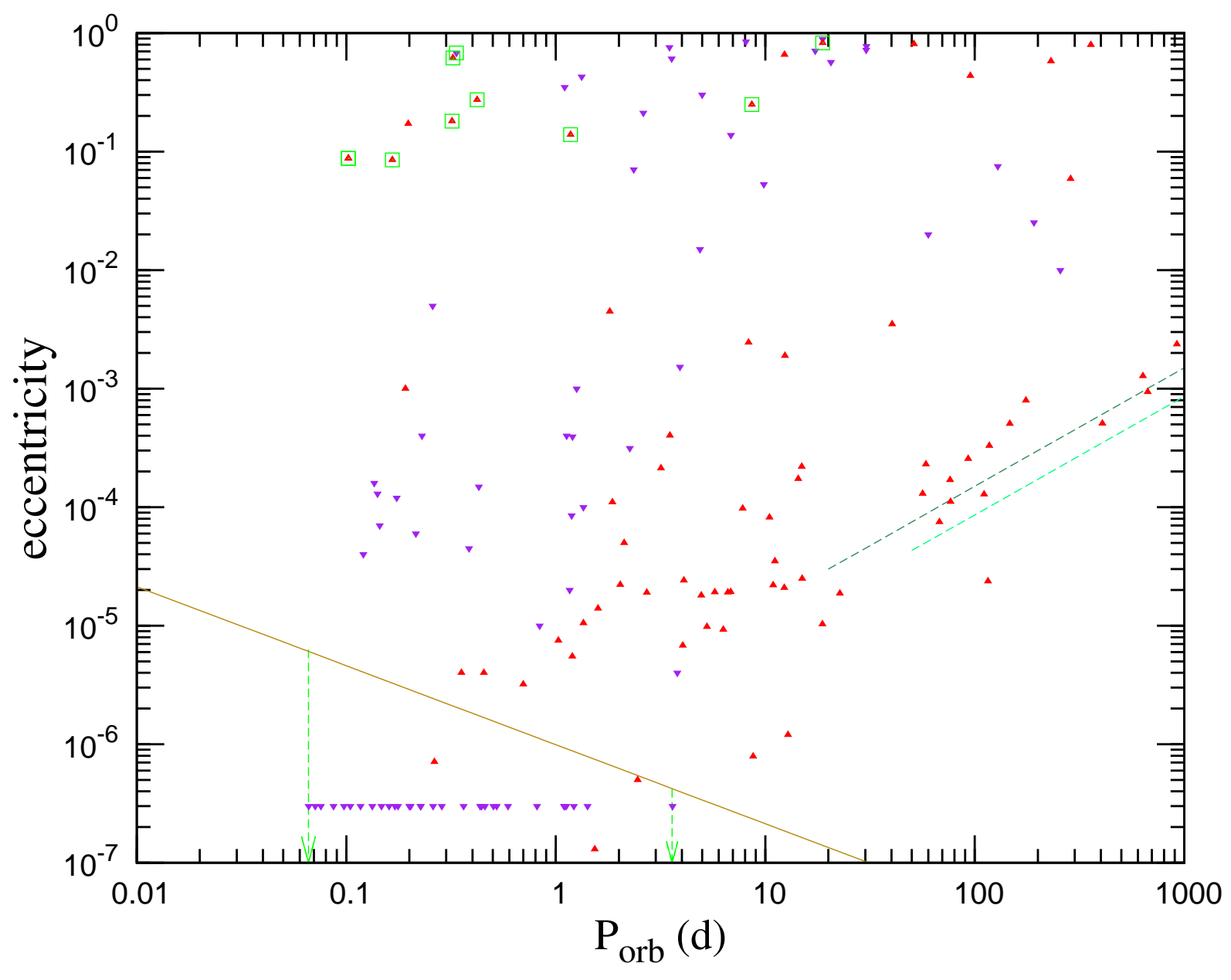

Fig. 1.- Plot of eccentricity vs orbital periods for binary radio pulsars in and out globular clusters. Purple $\mathbf{\nabla} \mathrm{s}$ are for globular cluster binaries and red $\boldsymbol{\Delta} \mathrm{s}$ are for disk binaries. The solid line in the lower left corner is the line for "limit of timing sensitivity". The dashed lines in the right side represents the eccentricity-orbital period relation as predicted by Phinney (1992) - the upper line is the original line drawn by Phinney (1992) and the lower line is using population II models. Double neutron star binaries are enclosed by green $\square$ s. 

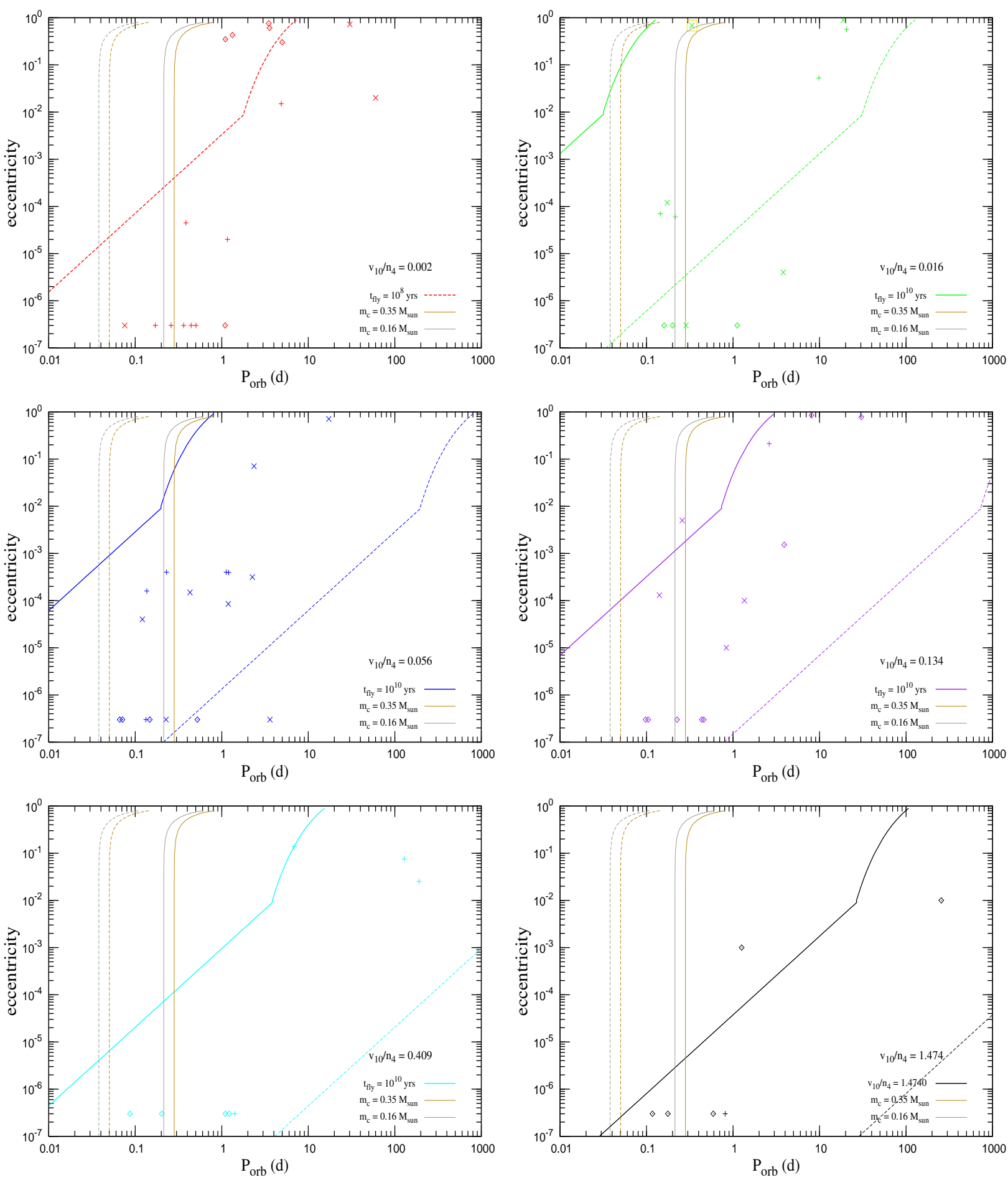

Fig. 2.- Cluster binary pulsars in the $e-P_{\text {orb }}$ plane with contours of $t_{f l y}=10^{10}$ yrs (solid lines) and $t_{f l y}=10^{8}$ yrs (dashed lines) for each group. Contours of $t_{g r}=10^{10}$ yrs (solid lines) and $t_{g r}=10^{8} \mathrm{yrs}$ (dashed lines) for binaries with $m_{p}=1.4 M_{\odot}$ and $m_{c}=0.35 M_{\odot}$ or $0.16 M_{\odot}$ are also shown. Pulsars with projected positions inside the cluster core are marked with + , those outside the cluster core are marked with $\times$ and the pulsars with unknown position offsets are marked with $\diamond$. Solid red line corresponding to $t_{f l y}=10^{10}$ years for $v_{10} / n_{4}=0.0024$ is outside the range plotted. Individual pulsars are marked with the same colors as the $v_{10} / n_{4}$ values of the host GCs. 

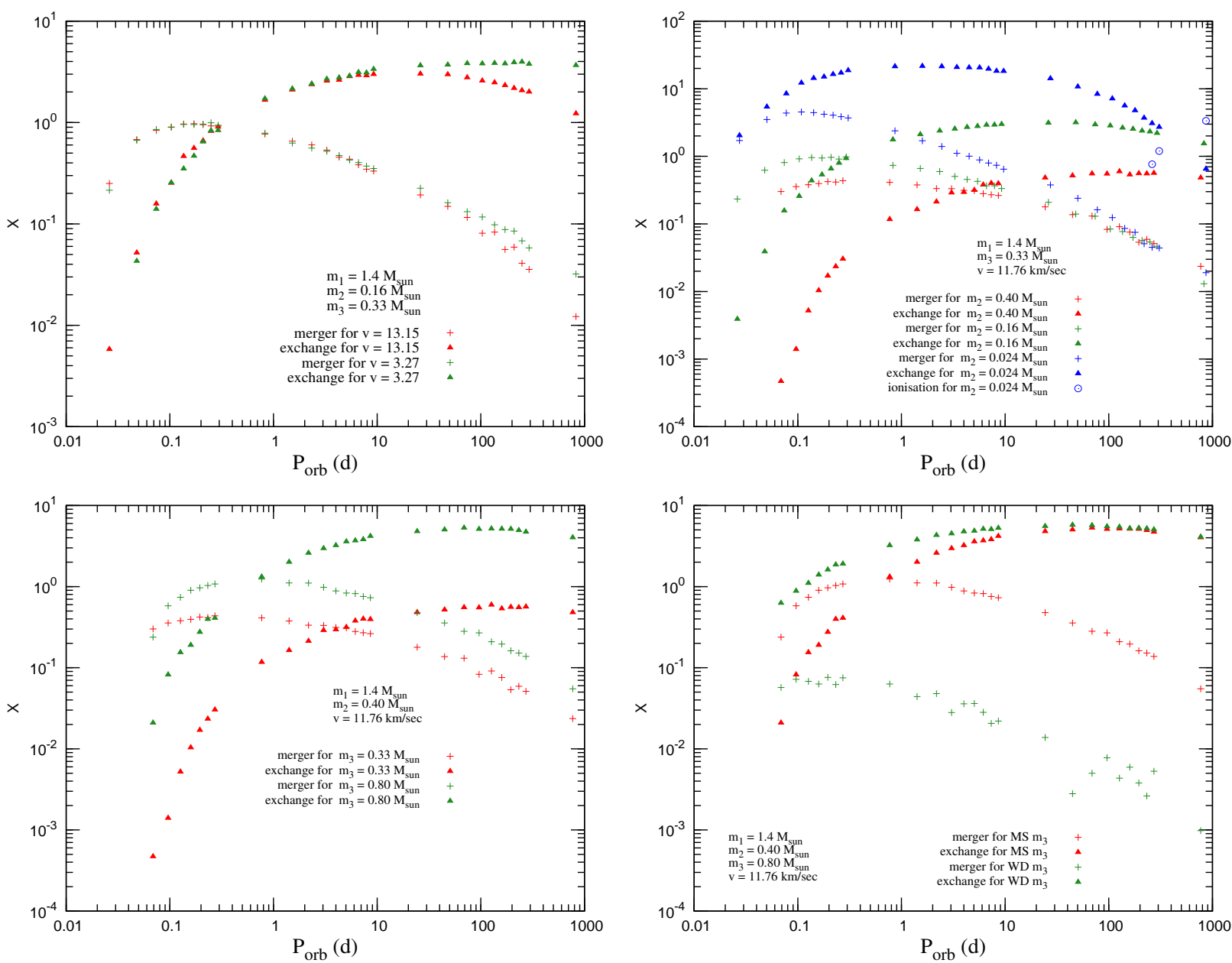

Fig. 3.- Variation of $X$ for exchange $(+)$, merger $(\boldsymbol{\Delta})$ and ionization (o, whenever significant) with $P_{\text {orb }, \text { in }}$. (i) The first figure is for $m_{1}=1.4 M_{\odot}, m_{2}=0.16 M_{\odot}, m_{3}=0.33 M_{\odot}$ and two values of $v$ e.g $13.16 \mathrm{~km} / \mathrm{sec}$ (red) and $3.27 \mathrm{~km} / \mathrm{sec}$ (green). (ii) The second figure is for $m_{1}=1.4 M_{\odot}, m_{3}=0.33 M_{\odot}, v=11.76 \mathrm{~km} / \mathrm{sec}$ and different values of $m_{2}$ e.g $0.40 M_{\text {odor }}$ (red), $0.16 M_{\odot}$ (green) and $0.024 M_{\odot}$ (blue). (iii) The third figure is for $m_{1}=1.4 M_{\odot}, m_{2}=0.16 M_{\odot}, v=11.76 \mathrm{~km} / \mathrm{sec}$ and different values of $m_{3}$ e.g. $0.33 M_{\odot}(\mathrm{red})$, $0.80 M_{\odot}$ (green). (iv) The fourth figure is for $m_{1}=1.4 M_{\odot}, m_{2}=0.16 M_{\odot}, m_{3}=0.80 M_{\odot}$, $v=11.76 \mathrm{~km} / \mathrm{sec}$ and the $3^{\text {rd }}$ star is either a MS (red) or a WD (green). 

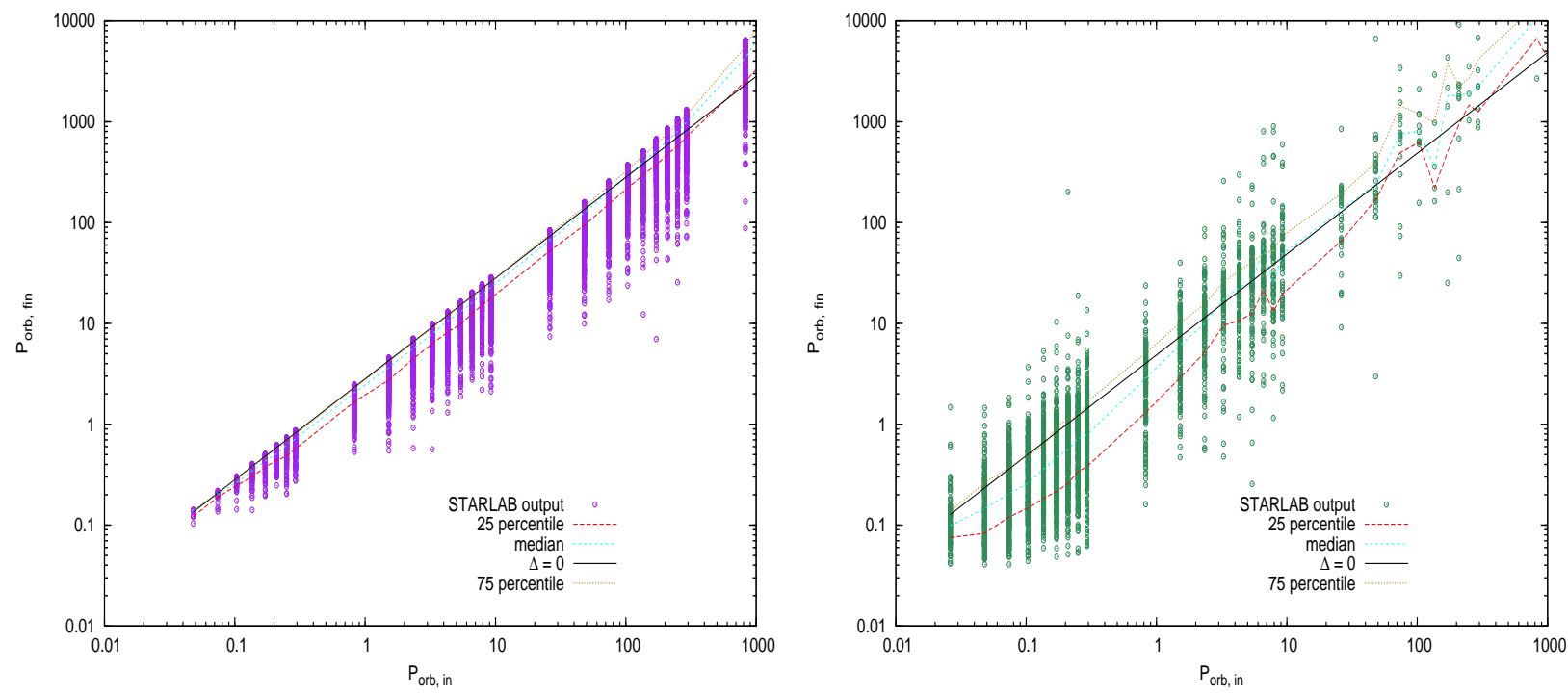

Fig. 4.- $P_{\text {orb, in }}$ Vs $P_{\text {orb, fin }}$ plots. Right hand side (purple points) diagram is for exchanges and the left hand side diagram (green points) is for mergers. The stellar parameters are as follows : $m_{1}=1.4 M_{\odot}(\mathrm{NS}), m_{3}=0.33 M_{\odot}(\mathrm{MS}), m_{2}=0.16 M_{\odot}(\mathrm{MS})$ and $v=11.76$ $\mathrm{km} / \mathrm{sec}$. 

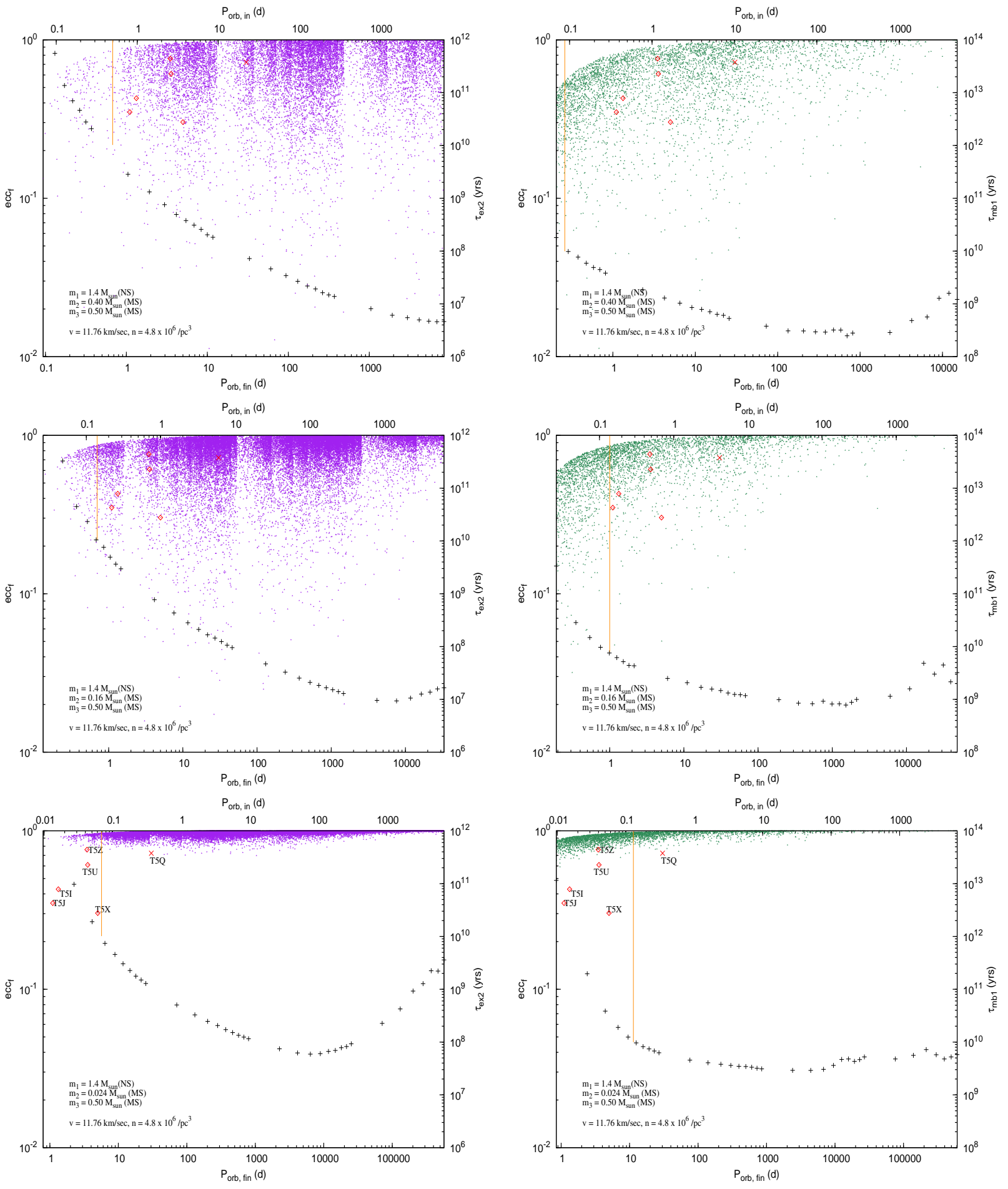

Fig. 5.- Time scales (denoted by ' + ') and final eccentricity distributions (scatter-plot of points) with initial and final orbital periods ( $\Delta=0$ in eq. 8) for exchange (red points: "exchange2") and merger (green points: "merger_ b1") interactions with different stellar parameters. We plot $P_{\text {orb }, \text { in }}$ along the top x-axis and $P_{\text {orb,fin }}$ along the bottom x-axis. The left y axis gives the final eccentricities while the right $\mathrm{y}$ axis gives the time scales of interactions. The vertical orange lines form the boundaries of the allowed orbital period regions where interaction time scales are less than $10^{10} \mathrm{yrs}$. The stellar parameters are as follows : $m_{1}=1.4 M_{\odot}, m_{3}=0.50 M_{\odot}$ and $m_{2}$ varies from $0.024 M_{\odot}$ to $0.16 M_{\odot}$ and then to $0.40 M_{\odot}$. 


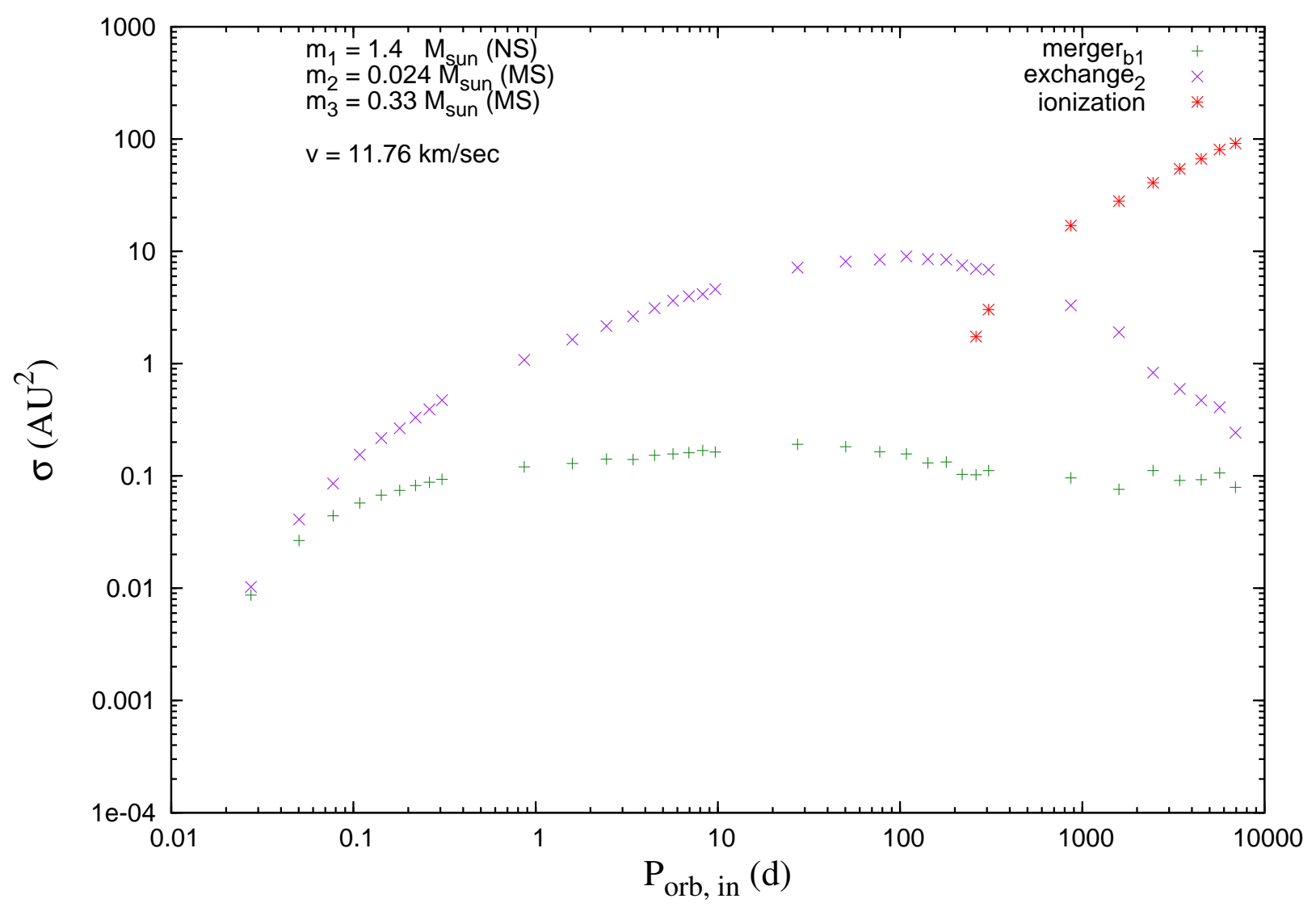

Fig. 6. - Variation of $\sigma$ for exchange (purple ' $\mathrm{x}$ '), merger (green ' + ') and ionization (red '*') with $P_{\text {orb, in }}$ for $m_{1}=1.4 M_{\odot}, m_{2}=0.024 M_{\odot}, m_{3}=0.33 M_{\odot}$ and $v=11.76 \mathrm{~km} / \mathrm{sec}$. 
Table 1: STARLAB runs with neutron star mass $m_{1}=1.40 M_{\odot}$ and radius $R_{1}=10 \mathrm{~km}$ throughout for $v=11.76 \mathrm{~km} / \mathrm{sec}$.

\begin{tabular}{|l|l|c|c|c|}
\hline Set & $\begin{array}{l}m_{1}, m_{2}, m_{3} \\
\left(M_{\odot}\right)\end{array}$ & $\begin{array}{c}R_{2}, R_{3} \\
\left(R_{\odot}\right)\end{array}$ & $v_{f} / v_{c}$ & $\begin{array}{c}P_{\text {orb }, \text { in }} \\
(\text { day })\end{array}$ \\
\hline 1 & $1.40,0.40,0.33$ & $0.40(\mathrm{MS}), 0.33(\mathrm{MS})$ & {$[0.018,0.788]$} & {$[0.07,6159]$} \\
2 & $1.40,0.16,0.33$ & $0.16(\mathrm{MS}), 0.33(\mathrm{MS})$ & {$[0.019,1.231]$} & {$[0.03,6616]$} \\
3 & $1.40,0.024,0.33$ & $0.024(\mathrm{MS}), 0.33(\mathrm{MS})$ & {$[0.035,3.153]$} & {$[0.01,6924]$} \\
4 & $1.40,0.40,0.80$ & $0.40(\mathrm{MS}), 0.80(\mathrm{MS})$ & {$[0.025,1.110]$} & {$[0.07,6159]$} \\
5 & $1.40,0.40,0.80$ & $0.40(\mathrm{MS}), 0.01(\mathrm{WD})$ & {$[0.025,1.110]$} & {$[0.07,6159]$} \\
6 & $1.40,0.16,0.80$ & $0.16(\mathrm{MS}), 0.80(\mathrm{MS})$ & {$[0.027,1.716]$} & {$[0.03,6616]$} \\
7 & $1.40,0.16,0.80$ & $0.16(\mathrm{MS}), 0.01(\mathrm{WD})$ & {$[0.027,1.716]$} & {$[0.03,6616]$} \\
8 & $1.40,0.024,0.80$ & $0.024(\mathrm{MS}), 0.80(\mathrm{MS})$ & {$[0.049,4.360]$} & {$[0.01,6924]$} \\
9 & $1.40,0.024,0.80$ & $0.024(\mathrm{MS}), 0.01(\mathrm{MS})$ & {$[0.049,4.360]$} & {$[0.01,6924]$} \\
10 & $1.40,0.40,0.50$ & $0.40(\mathrm{MS}), 0.50(\mathrm{MS})$ & {$[0.020,0.933]$} & {$[0.07,6159]$} \\
11 & $1.40,0.16,0.50$ & $0.16(\mathrm{MS}), 0.50(\mathrm{MS})$ & {$[0.023,1.452]$} & {$[0.03,6616]$} \\
12 & $1.40,0.024,0.50$ & $0.024(\mathrm{MS}), 0.50(\mathrm{MS})$ & {$[0.041,3.706]$,} & {$[0.01,6924]$} \\
\hline
\end{tabular}


Table 2: Globular cluster parameters taken from Webbink (1985).

\begin{tabular}{cccccc}
\hline group & GC & $\begin{array}{c}\text { d (from sun }) \\
(\mathrm{kpc})\end{array}$ & $\begin{array}{c}v_{10} \\
(10 \mathrm{~km} / \mathrm{sec})\end{array}$ & $\begin{array}{c}n_{4} \\
\left(10^{4} \mathrm{pc}^{-3}\right)\end{array}$ & $v_{10} / n_{4}$ \\
\hline 1 & Ter 5 & 10.3 & 1.18 & 479.77 & 0.0024 \\
\hline 2 & NGC 6440 & 8.4 & 1.30 & 102.10 & 0.013 \\
& M 30 & 8.0 & 0.52 & 36.39 & 0.014 \\
& NGC 1851 & 12.1 & 0.98 & 60.40 & 0.016 \\
& M 62 & 6.9 & 0.52 & 32.36 & 0.016 \\
& M 15 & 10.3 & 0.86 & 36.65 & 0.023 \\
\hline 3 & NGC 6441 & 11.2 & 1.44 & 31.70 & 0.046 \\
& NGC 6544 & 2.7 & 0.59 & 9.75 & 0.060 \\
& 47 Tuc & 4.5 & 1.32 & 31.99 & 0.062 \\
\hline 4 & M 28 & 5.6 & 1.06 & 9.59 & 0.110 \\
& NGC 6342 & 8.6 & 0.45 & 3.49 & 0.130 \\
& NGC 6752 & 4.0 & 0.78 & 5.97 & 0.131 \\
& NGC 6760 & 7.4 & 0.58 & 4.17 & 0.138 \\
& NGC 6539 & 8.4 & 0.45 & 3.09 & 0.146 \\
& NGC 6397 & 2.3 & 0.48 & 3.16 & 0.152 \\
\hline 5 & M 4 & 2.2 & 0.51 & 1.63 & 0.32 \\
& M 5 & 7.5 & 0.84 & 2.57 & 0.328 \\
& M 3 & 10.4 & 0.82 & 1.44 & 0.568 \\
& M 22 & 3.2 & 0.83 & 1.96 & 0.423 \\
\hline 6 & M 71 & 4.0 & 0.33 & 0.35 & 0.934 \\
& M 13 & 30.4 & 0.78 & 0.72 & 1.082 \\
& NGC 6749 & 7.9 & 0.39 & 0.23 & 1.713 \\
& M 53 & 17.8 & 0.65 & 0.30 & 2.167 \\
\hline
\end{tabular}


Table 3: Parameters for 73 binary pulsars with known orbital solutions in 23 globular clusters. Pulsar offsets from the cluster cores are in the units of the core radius $r_{c}$ (taken from Freire's webpage at http://www.naic.edu/ pfreire/GCpsr.html and S. Ransom's webpage at http://www.cv.nrao.edu/ sransom/). In Freire's webpage, eccentricities of 32 pulsars are listed as zero, which we have replaced by an arbitrarily small value 0.0000003 (see text for details). Last column shows the median values of companion masses i.e. for inclination angle $i=60$ degree (again from Freire's webpage) taking pulsar masses as $1.35 M_{\odot}$. For some pulsars, better mass measurements are available from the measurement of rates of periastron advance or from the spectral analysis of the optical counterpart in case of PSR J1911-5958A. These pulsars are listed here : (i) 47Tuc H has $m_{p}=1.44 M_{\odot}, m_{c}=0.17 M_{\odot}$ (Freire et al. 2003) (ii) NGC 1851A has $m_{p}=1.35 M_{\odot}, m_{c}=1.103 M_{\odot}$ (Freire, Ransom \& Gupta 2007) (iii) M5B has $m_{p}=2.08 M_{\odot}, m_{c}=0.21 M_{\odot}$ (Freire, Wolszczan, van den Berg \& Hessels, 2008) (iv) Ter 5 I has $m_{p}=1.87 M_{\odot}, m_{c}=0.30 M_{\odot}$ (Ransom et al. 2005) (v) Ter $5 \mathrm{~J}$ has $m_{p}=1.73 M_{\odot}, m_{c}=0.47 M_{\odot}$ (Ransom et al. 2005) (vi) NGC $6440 \mathrm{~B}$ has $m_{p}=2.74 M_{\odot}, m_{c}=0.18 M_{\odot}$ (Freire et al. 2008) (vii) NGC $6441 \mathrm{~A}$ has $m_{p}=1.26 M_{\odot}, m_{c}=0.67 M_{\odot}$ (Freire et al. 2008) (viii) PSR J1911-5958A has $m_{p}=1.34 M_{\odot}, m_{c}=0.175 M_{\odot}$ (Bassa, van Kerkwijk, Koester \& Verbunt 2006) (using spectral analysis of the optical counterpart ) (ix) M15 C has $m_{p}=1.358 M_{\odot}, m_{c}=1.354 M_{\odot}$ (Jacoby et al. 2006). The eclipsing pulsars are marked with "(e)" which most probably have main sequence companions (unless the inclination angle is very close to $90^{\circ}$ ).

\begin{tabular}{|c|c|c|c|c|c|c|c|c|c|}
\hline No & $\overline{\mathrm{GC}}$ & Pulsar & $\begin{array}{l}\text { offset } \\
\text { (in } \\
\left.r_{c}\right)\end{array}$ & $\begin{array}{l}P_{s} \\
(\mathrm{~ms})\end{array}$ & $\begin{array}{l}\dot{P}_{s} \\
\left(10^{-20}\right. \\
\mathrm{sec} / \mathrm{sec})\end{array}$ & $\begin{array}{l}\mathrm{DM} \\
\left(\mathrm{cm}^{-3} \mathrm{pc}\right)\end{array}$ & $\begin{array}{l}P_{\text {orb }} \\
\text { (d) }\end{array}$ & $\overline{e e}$ & $\begin{array}{l}m_{c} \\
\left(M_{\odot}\right)\end{array}$ \\
\hline 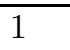 & 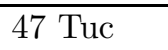 & "J0024-7205E & 1.477 & 3.536 & 9.851 & 24.23 & 2.25684 & 0.0003152 & 0.18 \\
\hline 2 & 47 Tuc & J0024-7204H & 1.75 & 3.210 & -0.183 & 24.36 & 2.35770 & 0.070560 & 0.19 \\
\hline 3 & 47 Tuc & J0024-7204I & 0.659 & 3.485 & -4.587 & 24.42 & 0.22979 & $<0.0004$ & 0.015 \\
\hline 4 & 47 Tuc & J0023-7203J (e) & 2.273 & 2.100 & -0.979 & 24.58 & 0.12066 & $<0.00004$ & 0.024 \\
\hline 5 & 47 Tuc & J0024-7204O & 0.136 & 2.643 & 3.035 & 24.36 & 0.13597 & $<0.00016$ & 0.025 \\
\hline 6 & 47 Tuc & J0024-7204P & $?$ & 3.643 & $?$ & 24.30 & 0.1472 & 0.0000003 & 0.02 \\
\hline 7 & 47 Tuc & J0024-7204Q & 2.227 & 4.033 & 3.402 & 24.29 & 1.18908 & 0.000085 & 0.21 \\
\hline 8 & 47 Tuc & J0024-7204R (e) & $?$ & 3.480 & $?$ & 24.40 & 0.0662 & 0.0000003 & 0.030 \\
\hline 9 & 47 Tuc & J0024-7204S & 0.432 & 2.830 & -12.054 & 24.35 & 1.20172 & 0.000394 & 0.10 \\
\hline 10 & 47 Tuc & J0024-7204T & 0.773 & 7.588 & 29.37 & 24.39 & 1.12618 & 0.00040 & 0.20 \\
\hline 11 & 47 Tuc & J0024-7203U & 2.136 & 4.343 & 9.523 & 24.33 & 0.42911 & 0.000149 & 0.14 \\
\hline 12 & 47 Tuc & J0024-7204V (e?) & $?$ & 4.810 & $?$ & 24.10 & 0.227 & 0.0000003 & 0.35 \\
\hline 13 & 47 Tuc & J0024-7204W (e) & 0.182 & 2.352 & $?$ & 24.30 & 0.1330 & 0.0000003 & 0.14 \\
\hline 14 & 47 Tuc & J0024-7204Y & $?$ & 2.197 & $?$ & 24.20 & 0.52194 & 0.0000003 & 0.16 \\
\hline 15 & NGC1851 & J0514-4002A & 1.333 & 4.990 & 0.117 & 52.15 & 18.78518 & 0.8879773 & 1.10 \\
\hline 16 & M53 & $\mathrm{B} 1310+18$ & $?$ & 33.163 & $?$ & 24.00 & 255.8 & 0.01 & 0.35 \\
\hline 17 & M3 & $\mathrm{J} 1342+2822 \mathrm{~B}$ & 0.254 & 2.389 & 1.858 & 26.15 & 1.41735 & 0.0000003 & 0.21 \\
\hline 18 & M3 & $\mathrm{J} 1342+2822 \mathrm{D}$ & 0.418 & 5.443 & $?$ & 26.34 & 128.752 & 0.0753 & 0.21 \\
\hline
\end{tabular}


Table 3: (continued).

\begin{tabular}{|c|c|c|c|c|c|c|c|c|c|}
\hline "No & 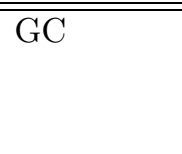 & Pulsar & $\begin{array}{l}\text { offset } \\
\text { (in } \\
\left.r_{c}\right)\end{array}$ & $\begin{array}{l}P_{s} \\
(\mathrm{~ms})\end{array}$ & $\begin{array}{l}\dot{P}_{s} \\
\left(10^{-20}\right. \\
\sec / \mathrm{sec})\end{array}$ & $\begin{array}{l}\mathrm{DM} \\
\left(\mathrm{cm}^{-3} \mathrm{pc}\right)\end{array}$ & $\begin{array}{l}P_{\text {orb }} \\
\text { (d) }\end{array}$ & 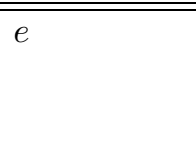 & $\begin{array}{l}m_{c} \\
\left(M_{\odot}\right)\end{array}$ \\
\hline 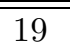 & M5 & 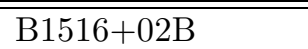 & "0.545 & 7.947 & "-0.331 & 29.45 & 6.85845 & 0.13784 & "0.13 \\
\hline 20 & M5 & $\mathrm{J} 1518+0204 \mathrm{C}(\mathrm{e})$ & $?$ & 2.484 & $?$ & 29.30 & 0.087 & 0.0000003 & 0.038 \\
\hline 21 & M5 & $\mathrm{J} 1518+0204 \mathrm{D}$ & $?$ & 2.988 & $?$ & 29.30 & 1.22 & 0.0000003 & 0.20 \\
\hline 22 & M5 & $\mathrm{J} 1518+0204 \mathrm{E}$ & $?$ & 3.182 & $?$ & 29.30 & 1.10 & 0.0000003 & 0.15 \\
\hline 23 & M4 & B1620-26 & 0.924 & 11.076 & -5.469 & 62.86 & 191.44281 & 0.02531545 & 0.33 \\
\hline 24 & M13 & $\mathrm{B} 1639+36 \mathrm{~B}$ & $?$ & 3.528 & $?$ & 29.50 & 1.25911 & $<0.001$ & 0.19 \\
\hline 25 & M13 & $\mathrm{J} 1641+3627 \mathrm{D}$ & $?$ & 3.118 & $?$ & 30.60 & 0.591 & 0.0000003 & 0.18 \\
\hline 26 & M13 & $\mathrm{J} 1641+3627 \mathrm{E}(\mathrm{e} ?)$ & $?$ & 2.487 & $?$ & 30.30 & 0.117 & 0.0000003 & 0.02 \\
\hline 27 & M62 & J1701-3006A & 1.778 & 5.241 & -13.196 & 115.03 & 3.80595 & 0.000004 & 0.23 \\
\hline 28 & M62 & J1701-3006B (e) & 0.155 & 3.594 & -34.978 & 113.44 & 0.14455 & $<0.00007$ & 0.14 \\
\hline 29 & M62 & J1701-3006C & 0.972 & 3.806 & -3.189 & 114.56 & 0.21500 & $<0.00006$ & 0.08 \\
\hline 30 & M62 & J1701-3006D & $?$ & 3.418 & $?$ & 114.31 & 1.12 & 0.0000003 & 0.14 \\
\hline 31 & M62 & J1701-3006E (e) & $?$ & 3.234 & $?$ & 113.78 & 0.16 & 0.0000003 & 0.035 \\
\hline 32 & M62 & $\mathrm{J} 1701-3006 \mathrm{~F}$ & $?$ & 2.295 & $?$ & 113.36 & 0.20 & 0.0000003 & 0.02 \\
\hline 33 & NGC6342 & B1718-19 (e) & 46.000 & 1004.04 & $1.59 \times 10^{5}$ & 71.00 & 0.25827 & $<0.005$ & 0.13 \\
\hline 34 & NGC6397 & $\mathrm{J} 1740-5340(\mathrm{e})$ & 18.340 & 3.650 & 16.8 & 71.80 & 1.35406 & $<0.0001$ & 0.22 \\
\hline 35 & Ter5 & J1748-2446A (e) & 2.778 & 11.5632 & -3.400 & 242.10 & 0.075646 & 0.0000003 & 0.10 \\
\hline 36 & Ter5 & J1748-2446E & out(1.6) & 2.19780 & $?$ & 236.84 & 60.06 & 0.02 & 0.25 \\
\hline 37 & Ter5 & J1748-2446I & $?$ & 9.57019 & $?$ & 238.73 & 1.328 & 0.428 & 0.24 \\
\hline 38 & Ter5 & J1748-2446J & $?$ & 80.3379 & $?$ & 234.35 & 1.102 & 0.350 & 0.39 \\
\hline 39 & Ter5 & J1748-2446M & $\operatorname{in}(0.48)$ & 3.56957 & $?$ & 238.65 & 0.4431 & 0.0000003 & 0.16 \\
\hline 40 & Ter5 & $\mathrm{J} 1748-2446 \mathrm{~N}$ & $\operatorname{in}(0.39)$ & 8.66690 & $?$ & 238.47 & 0.3855 & 0.000045 & 0.56 \\
\hline 41 & Ter5 & $\mathrm{J} 1748-2446 \mathrm{O}(\mathrm{e})$ & $\operatorname{in}(0.45)$ & 1.67663 & $?$ & 236.38 & 0.2595 & 0.0000003 & 0.04 \\
\hline 42 & Ter5 & $\mathrm{J} 1748-2446 \mathrm{P}(\mathrm{e})$ & $\operatorname{in}(0.74)$ & 1.72862 & $?$ & 238.79 & 0.3626 & 0.0000003 & 0.44 \\
\hline 43 & Ter5 & J1748-2446Q & $\operatorname{out}(1.45)$ & 2.812 & $?$ & 234.50 & 30.295 & 0.722 & 0.53 \\
\hline 44 & Ter5 & $\mathrm{J} 1748-2446 \mathrm{U}$ & $?$ & 3.289 & $?$ & 235.46 & 3.57 & 0.61 & 0.46 \\
\hline 45 & Ter5 & J1748-2446V & $\operatorname{in}(0.90)$ & 2.07251 & $?$ & 239.11 & 0.5036 & 0.0000003 & 0.14 \\
\hline 46 & Ter5 & J1748-2446W & $\operatorname{in}(0.42)$ & 4.20518 & $?$ & 239.14 & 4.877 & 0.015 & 0.34 \\
\hline 47 & Ter5 & J1748-2446X & $?$ & 2.99926 & $?$ & 240.03 & 4.99850 & 0.3024 & 0.29 \\
\hline 48 & Ter5 & J1748-2446Y & $\operatorname{in}(0.55)$ & 2.04816 & $?$ & 239.11 & 1.16443 & 0.00002 & 0.16 \\
\hline 49 & Ter5 & J1748-2446Z & $?$ & 2.46259 & $?$ & 238.85 & 3.48807 & 0.7608 & 0.25 \\
\hline 50 & Ter5 & J1748-2446ad (e) & $?$ & 1.39595 & $?$ & 235.60 & 1.09443 & 0.0000003 & 0.16 \\
\hline 51 & Ter5 & J1748-2446ae & $\operatorname{in}(0.42)$ & 3.65859 & $?$ & 238.75 & 0.17073 & 0.0000003 & 0.019 \\
\hline 52 & NGC6440 & J1748-2021B & 0.530 & 16.760 & -32.913 & 220.92 & 20.550 & 0.570 & 0.090 \\
\hline 53 & NGC6440 & J1748-2021D (e) & 4.230 & 13.496 & 58.678 & 224.98 & 0.286 & 0.0000003 & 0.14 \\
\hline 54 & NGC6440 & $\mathrm{J} 1748-2021 \mathrm{~F}$ & 0.690 & 3.794 & 31.240 & 224.10 & 9.83397 & 0.0531 & 0.35 \\
\hline 55 & NGC6441 & J1750-37A & 1.910 & 111.609 & 566.1 & 233.82 & 17.3 & 0.71 & 0.70 \\
\hline 56 & NGC6441 & J1750-3703B & 3.000 & 6.074 & 1.92 & 234.39 & 3.61 & 0.0000003 & 0.19 \\
\hline 57 & NGC6539 & B1802-07 & 0.463 & 23.1009 & 47.0 & 186.38 & 2.61676 & 0.21206 & 0.35 \\
\hline 58 & NGC6544 & B1802-07 & $?$ & 3.05945 & $?$ & 134.0 & 0.071092 & 0.0000003 & 0.010 \\
\hline
\end{tabular}


Table 3: (continued).

\begin{tabular}{|c|c|c|c|c|c|c|c|c|c|}
\hline No & GC & Pulsar & $\begin{array}{l}\text { offset } \\
\text { (in } \\
\left.r_{c}\right) \\
\end{array}$ & $\begin{array}{l}P_{s} \\
(\mathrm{~ms}) \\
\end{array}$ & $\begin{array}{l}\dot{P}_{s} \\
\left(10^{-20}\right. \\
\mathrm{sec} / \mathrm{sec})\end{array}$ & $\begin{array}{l}\mathrm{DM} \\
\left(\mathrm{cm}^{-3} \mathrm{pc}\right)\end{array}$ & $\begin{array}{l}P_{\text {orb }} \\
\text { (d) } \\
\end{array}$ & $e$ & $\begin{array}{l}m_{c} \\
\left(M_{\odot}\right) \\
\end{array}$ \\
\hline 59 & M28 & J1824-2452C & $?$ & 4.159 & $?$ & 120.70 & 8.078 & 0.847 & 0.30 \\
\hline 60 & M28 & J1824-2452D & $?$ & 79.832 & $?$ & 119.50 & 30.404 & 0.776 & 0.45 \\
\hline 61 & M28 & J1824-2452G & $?$ & 5.909 & $?$ & 119.40 & 0.1046 & 0.0000003 & 0.011 \\
\hline 62 & M28 & J1824-2452H (e) & $?$ & 4.629 & $?$ & 121.50 & 0.435 & 0.0000003 & 0.20 \\
\hline 63 & M28 & J1824-2452I (e) & $?$ & 3.93185 & $?$ & 119.00 & 0.45941 & 0.0000003 & 0.20 \\
\hline 64 & M28 & J1824-2452J & $?$ & 4.039 & $?$ & 119.20 & 0.0974 & 0.0000003 & 0.015 \\
\hline 65 & M28 & J1824-2452K & ? & 4.46105 & ? & 119.80 & 3.91034 & 0.001524 & 0.16 \\
\hline 66 & M28 & J1824-2452L & $?$ & 4.10011 & $?$ & 119.00 & 0.22571 & 0.0000003 & 0.022 \\
\hline 67 & M22 & J1836-2354A & $?$ & 3.35434 & $?$ & 89.10 & 0.20276 & 0.0000003 & 0.020 \\
\hline 68 & NGC6749 & $\mathrm{J} 1905+0154 \mathrm{~A}$ & 0.662 & 3.193 & $?$ & 193.69 & 0.81255 & 0.0000003 & 0.090 \\
\hline 69 & NGC6752 & J1911-5958A & 37.588 & 3.26619 & 0.307 & 33.68 & 0.83711 & $<0.00001$ & 0.22 \\
\hline 70 & NGC6760 & $\mathrm{J} 1911+0102 \mathrm{~A}$ & 1.273 & 3.61852 & -0.658 & 202.68 & 0.140996 & $<0.00013$ & 0.020 \\
\hline 71 & M71 & J1953+1846A (e) & $?$ & 4.888 & $?$ & 117.00 & 0.1766 & 0.0000003 & 0.032 \\
\hline 72 & M15 & $\mathrm{B} 2127+11 \mathrm{C}$ & 13.486 & 30.5293 & 499.1 & 67.13 & 0.33528 & 0.681386 & 1.13 \\
\hline 73 & M30 & J2140-2310A (e) & 1.117 & 11.0193 & -5.181 & 25.06 & 0.17399 & $<0.00012$ & 0.11 \\
\hline
\end{tabular}

